\title{
Title: Secondary ossification center induces and protects growth plate structure
}

Authors: Meng Xie ${ }^{1}$, Pavel Gol'din², Anna Nele Herdina ${ }^{1,19}$, Jordi Estefa ${ }^{3}$, Ekaterina V Medvedeva ${ }^{4}$, Lei $\mathrm{Li}^{1}$, Phillip T Newton ${ }^{1,5}$, Svetlana Kotova ${ }^{4,6}$, Boris Shavkuta, ${ }^{4,7}$ Aditya Saxena ${ }^{8}$, Lauren T Shumate ${ }^{9}$, Brian Metscher ${ }^{10}$, Karl Großschmidt ${ }^{11}$, Shigeki Nishimori ${ }^{9}$, Anastasia Akovantseva ${ }^{4,7}$, Anna P Usanova ${ }^{4}$, Anastasiia D Kurenkova ${ }^{4}$, Anoop Kumar ${ }^{15}$, Irene Linares Arregui ${ }^{12}$, Paul Tafforeau ${ }^{13}$, Kaj Fried ${ }^{14}$, Mattias Carlström ${ }^{1}$, Andras Simon ${ }^{15}$, Christian Gasser $^{12}$, Henry M Kronenberg ${ }^{9}$, Murat Bastepe ${ }^{9}$, Kimberly L. Cooper ${ }^{8}$, Peter Timashev ${ }^{4,6,7,16}$, Sophie Sanchez ${ }^{3,13,17}$, Igor Adameyko ${ }^{1,18}$, Anders Eriksson ${ }^{19}$, Andrei S Chagin ${ }^{1,4^{*}}$

\section{Affiliations}

1. Department of Physiology and Pharmacology, Karolinska Institutet, Stockholm, Sweden

2. Department of Evolutionary Morphology, Schmalhausen Institute of Zoology of NAS of Ukraine, Ukraine

3. Science for Life Laboratory and Uppsala University, Subdepartment of Evolution and Development, Department of Organismal Biology, Uppsala, Sweden

4. Institute for Regenerative Medicine, Sechenov University, Russia

5. Department of Women's and Children's Health, Astrid Lindgren Children's Hospital, Karolinska University Hospital, Sweden

6. Semenov Institute of Chemical Physics, Russia

7. Institute of Photonic Technologies, Research center "Crystallography and Photonics", Russia

8. Division of Biological Sciences, University of California San Diego, USA

9. Endocrine Unit, Department of Medicine, Massachusetts General Hospital and Harvard Medical School, Boston, U.S.A.

10. Department of Theoretical Biology, University of Vienna, Austria

11. Bone and Biomaterials Research, Center for Anatomy and Cell Biology, Medical University of Vienna, Vienna, Austria

12. Department of Solid Mechanics, KTH Royal Institute of Technology, Stockholm, Sweden

13. European Synchrotron Radiation Facility, Grenoble Cedex, France

14. Department of Neuroscience, Karolinska Institutet, Stockholm, Sweden

15. Department of Cell and Molecular Biology, Centre of Developmental Biology for Regenerative Medicine, Karolinska Institute, Stockholm, Sweden

16. Chemistry Department, Lomonosov Moscow State University, Leninskiye Gory 1-3, Moscow 119991, Russian Federation

17. Sorbonne Université - CR2P - MNHN, CNRS, UPMC, Paris, France

18. Department of Molecular Neurosciences, Medical University of Vienna, Austria

19. Department of Mechanics, KTH Royal Institute of Technology, Stockholm, Sweden

20. Division of Anatomy, MIC, Medical University of Vienna, Austria

*Corresponding author:

Andrei S Chagin andrei.chagin@ki.se 


\begin{abstract}
Growth plate and articular cartilage constitute a single anatomical entity early in development, but later separate into two distinct structures by the secondary ossification center (SOC). The reason for such separation remains unknown. We found that evolutionarily SOC appears in animals conquering the land - amniotes. Analysis of ossification pattern in mammals with specialized extremities (whales, bats, jerboa) revealed that SOC development correlates with the extent of mechanical loads. Mathematical modelling revealed that SOC reduces mechanical stress within the growth plate. Functional experiments revealed high vulnerability of hypertrophic chondrocytes to mechanical stress and showed that SOC protects these cells from apoptosis caused by extensive loading. Atomic force microscopy showed that hypertrophic chondrocytes are the least mechanically stiff cells within the growth plate. Altogether, these findings suggest that SOC has evolved to protect the hypertrophic chondrocytes from the high mechanical stress encountered in the terrestrial environment.
\end{abstract}

\title{
Introduction
}

The mammalian skeleton articulates via articular cartilage, covering the ends of long bones, and grows in length via epiphyseal cartilage, presented as tiny discs of chondrocytes referred to as growth plates and located near the ends of long bones. The growth plate chondrocytes proliferate, align in the longitudinal direction and then undergo several-fold enlargement (hypertrophy). Thereafter, the hypertrophic chondrocytes undergo apoptosis or transdifferentiation (1), leaving their calcified extracellular matrix as a scaffold on which invading blood vessels and osteoblasts form new bone tissue, a process known as endochondral bone formation. The process of endochondral bone formation predominantly depends on chondrocyte hypertrophy, and is very conserved throughout evolution of vertebrates, being described for fin-rayed fish, lobe-finned fish, stem tetrapods and modern humans (2-4).

In humans and most experimental models commonly employed to study bone growth (i.e., mice, rats and rabbits), the growth plate is separated from the articular cartilage by a bony fragment, called a bony epiphysis, generated developmentally from a secondary ossification center (SOC). In rodents and humans, this skeletal element forms during early postnatal development and splits the initially contiguous cartilaginous element (the cartilaginous epiphysis) into the distinct articular and growth plate cartilage (2) (see Figure 1figure supplement 1A-B for orientation). Interestingly, this spatial separation of articular and growth plate structures is not always required for articulation or bone growth, since it is absent in several vertebrate taxa, such as stem tetrapods (3,4), Chelonians, Crocodilians and Urodeles (salamanders) $(5,6)$ (see examples of salamander cartilage in Figure 1-figure supplement 1CD).

These observations raise the intriguing question as to why in many taxa including humans the cartilaginous epiphysis evolved into two functionally and spatially separated structures, one responsible for skeletal articulation and the other one required for skeletal growth. In light of the fact that both of these structures are involved in numerous pathologies (e.g., growth failure in children and osteoarthritis in seniors), answering this question may be of considerable clinical significance.

On the basis of an anatomical comparison between the epiphyses of different tetrapods, Haines proposed in 1942 that this separation evolved in evolution to improve the mechanical properties of the cartilaginous joint (7). An alternative hypothesis (based on mathematical modeling) suggested that the SOC not precedes, but is caused by mechanical 
stimulations (8). Here, we performed evolutionary and comparative analysis, mathematic modelling, physical tests and biological experiments, which altogether indicate that this separation evolved to protect the growth plate from high mechanical forces associated with weight-bearing in terrestrial environment.

\section{Results}

\section{Evolutionary analysis}

Confirming previous observations $(3,4)$, 3D microanatomical characterization of the 380-million-year-old lobe-finned fish Eusthenopteron revealed longitudinally-oriented trabeculae within the shaft of their humeri (Figure 1A), strongly indicating that endochondral ossification facilitates bone elongation in stem tetrapods in a manner similar to that in stem amniotes (290 Mya) (e.g., Seymouria, Figure 1B) and present-day mammals (e.g., red squirrel, Figure 1C).

Evolutionary analysis revealed that the SOC is not present in anamniotes and first appears in amniotes, animals which relocate their entire lifecycle on land (Figure 1D). Among various groups of synapsids, the SOC is present in all crown mammals, whereas some stem mammals have evolved a SOC [i.e., Niassodon (9) (Figure 1D)], but others have not (10, 11). In sauropsids, which can be subdivided into two large clades, Lepidosauria (lizards and Sphenodon) and Archosauria (crocodilians, chelonians, birds and non-avian dinosaurs), diversification of bone ossification is greater. Lepidosauria generally develop a SOC (lizards) or calcified secondary center (SC, sphenodontids) (Figure 1D). However Archosauria do not develop such structures [(6), Figure 1D]. Instead, this clade develops bone marrow protrusions with calcified or ossified walls that protrude into the epiphyseal cartilage and sequester the hypertrophic chondrocytes in between them $(6,7,12,13)$. These ossified protrusions are relatively small in chelonians and crocodilians and quite extended in birds and non-avian dinosaurs $((6,7,10,11)$ and Figure 1E). Although the weight-bearing bones of certain birds exhibit a SOC together with such protrusions (e.g., the proximal epiphysis of the tibiotarsus in sparrow (Figure 1E)), most ossify without forming a SOC (13).

It has to be emphasized that anamniotes spend their juvenile growth period in an aquatic environment, with amniotes being the first to translocate their entire life cycle on land. Such habitat change may pose additional mechanical demands on the growing skeleton, which must be sufficiently rigid to bear the body weight during a wide range of movements, yet flexible enough to allow bone elongation. Accordingly, we next hypothesized that development of the SOC or ossified protrusions might be viewed as skeletal adaptations to the weight-bearing demands of the terrestrial environment and associated mechanical stresses posed on the epiphyseal cartilage.

\section{Mathematical modeling}

To explore the potential effects of the SOC and ossified protrusions on stress distribution within the epiphyseal cartilage, we first employed mathematical modelling (i.e., finite element analysis, FEA) based on a modified model developed by Carter and co-workers $(8,14)$ (Figure $1 \mathrm{~F}$, see the methods section for details). For description of the local force effects in an equilibrium state, we evaluated the distributions within the model of maximum compressive principal stress, octahedral stress, and hydrostatic stress. These scalar point-wise stress state measures can in essence be seen as representing the local stress state by the dominant pressure in any direction (maximum compressive principal stress), the deforming stress tending to deform a spherical region into an ellipsoidal one (octahedral stress), and the uniform shapepreserving pressure tending to change just the size of a sphere (hydrostatic stress). In this 
144 evaluation, the shape-changing octahedral shear stress was provisionally assumed to be the

most significant one for chondrocytes (based on (8)).

First, the FEA showed that the SOC significantly enhances the stiffness of the entire epiphyseal structure (note the level of deflection in Figure 1F), thereby preventing severe distortion and consequent instability during locomotion. This provides experimental support to the idea proposed by Haines in 1942 (7). Furthermore, FEA revealed that the presence of either a SOC or ossified protrusions reduces the extent of octahedral shear stress distribution (associated with either a vertical or an angled load, the latter mimicking locomotion) within the region of epiphyseal plate, particularly where the hypertrophic chondrocytes are located (Figure $1 \mathrm{~F}$, see other stresses in Figure 1-figure supplement 2). Interestingly, despite being similarly protective for epiphyseal chondrocytes, the ossified protrusions do not improve the stiffness of the joint structure. Thus, our mathematical modelling indicates that both of these skeletal adaptations reduce octahedral stress within the epiphyseal cartilage, plausibly representing alternative evolutionary strategies for achieving the same goal.

Thus, in our subsequent analysis, we focused only on the SOC since it is most relevant to human physiology.

\section{Comparative analysis of animals with specialized extremities}

Thus, evolutionary analysis suggests that appearance of the SOC can be a skeletal adaptation to terrestrial environment, whereas mathematical modeling suggests that this adaptation may evolve to protect the growth plate structure from mechanical stresses. To further explore the association between mechanical demands and the extent of the SOC development, we selected a few extant unrelated taxa with extremities subjected to different mechanical demands, specifically Chiroptera (bats), Dipodoidea (Jerboa (i.e., bipedal hopping rodents)) and Cetaceans (whales).

\section{Chiroptera}

The newborn pups of Chiroptera must cling to their mother or to the roost from birth, utilizing for this purpose only their feet, thumbs of the wing and milk teeth, whereas other wing bones are not used until 2-3 weeks of age, when the pups learn to fly (15-18). We observed that in newborn bats SOC development in legs and thumbs of wings is more advanced than in the rest of wing bones (Figure 2A and Video 1). This heterochrony, i.e. change in timing or rate of ontogenetic development, was particularly pronounced in autopods with heavy ossified feet and thumb, but no SOC in other bones of the wing (Figure 2B-C). It was apparent during ontogenesis and consistent within the Chiroptera order (summary of 6 species with available ontogeny data is presented in Figure 2D-E and primary data for all 13 species analyzed presented in Supplementary File 1). It has to be admitted that despite a very clear pattern of heterochrony there is a variation of ossification among Chiroptera species, which likely reflects their varying foraging and roosting ecology, behavior of juvenile bats, litter size, and lifespan (19-23).

Interestingly, SOCs in the feet and the thumbs of some bat species are welldeveloped even before birth (Supplementary File 1) and the pups of certain species (Mops condylurus, Artibeus jamaicensis, Megaloglossus woermani and Rousettus celebensis) are born with fully ossified feet of adult size $(19,21)$ (see also Figure 2B for A. jamaicensis). The appearance of SOC in embryonic feet and thumb suggests that a genetic program governing SOC development precedes the mechanical influence.

\section{Jerboa}



jaculus) in the family of Dipodoidea rodents. These animals jumps on their hindlimbs when adult but during the first 2-3 weeks of life they employ only forelimbs thereafter becoming quadrupedal and eventually bipedal (24). The video-recording of gait pattern acquisition for two species Jacuslus Jaculus and Jaculus Orientalis confirmed that jerboa employs only front limbs for crawling during the first postnatal weeks (Video 2-3). Analysis of the ossification pattern for Jacuslus Jaculus revealed that SOC formation in the front limbs significantly preceded the one in the hind limbs (Figure 3A-R). It is interesting to point out that development of the SOC also coincides with the changes in gait pattern from crawling to walking in mice and rats (Figure 1-figure supplement 1A-B). Whether the same situation applies to human remains to be investigated.

Thus, in both Chiroptera and Jerboa, advanced ossification is observed in limbs

\section{Cetaceans}

If a demand for SOC is mechanically related, we further reasoned that the absence of terrestrial-associated loading conditions would release the evolutionary pressure on SOC, and that it can be lost or reduced with time. To test this assumption, we analyzed the cetaceans (whales) forelimbs (Figure 4A) whose mechanical demands highly differ in loadings and angles from their terrestrial ancestors (25). In early cetaceans from Eocene era (56-33.9 Mya) the epiphyseal plates of the forelimbs are structurally similar to those of terrestrial mammals, as evident in semi-aquatic Maiacetus inuus (26) and fully aquatic Dorudon atrox (27), where SOCs are present at the joints connecting carpal bones, metacarpal bones and phalanges (Figure 4B1-2). However, in baleen whales (both extant and some extinct from Miocene era, 23-5.3 Mya) there is a clear reduction of SOCs such as in the Balaenopteridae family where SOC is reduced by up to $80 \%$ in metacarpals and phalanges (Figure 4B3) and the Balaenidae family where SOC is reduced in distal radius and ulna and essentially absent in metacarpals phalanges (Figure 4B4).

Toothed whales from the Miocene era to the present demonstrate more diverse patterns of secondary ossification. SOCs are not reduced in either Platanistidae or certain Delphinoidea (Figure 4B5), whereas Iniidae (Amazon River dolphins) do not have distinct epiphyseal plates, but rather multiple small SOCs covering $10-50 \%$ of the corresponding area (Figure 4B6). In Ziphiidae and the narwhal Monodon monoceros (Delphinapteridae), SOCs are reduced in size by 5-10 times (Figure 4B7). X-ray examination of another member of Delphinapteridae, the beluga whale Delphinapterus leucas, revealed only remnants of SOC in metacarpals and phalanges (Figure 4D). Finally, the most advanced epiphyseal transformation is seen in the killer whale Orcinus orca (Delphinidae) (Figure 4B8) $(28,29)$ and the sperm whale Physeter catodon (Figure 4B9, 10), in which the SOCs of distal radius, ulna and metacarpals are indistinct or even absent and a fibrocartilaginous structure is formed in the carpal area (Figure 4B8-10). CT scan confirmed a complete lack of the SOC in Orcinus orca (Figure 4E and Video 4), which was further confirmed by radiograph (Figure 4C). Taken together, these observations suggest that the return of cetaceans to aquatic environment is associated with a gradual reduction in the size of SOC in a few phylogenetic lineages, and even their complete loss in some species.

The observed striking consistency in unrelated taxa indicates a causative relationship between the presence of the SOC and mechanical demands, and considering mathematical 
predictions and evolutionary analysis, further suggests that SOC protects the growth plate from mechanical demands.

\section{Functional experiments with two model systems: the SOC protects epiphyseal chondrocytes}

To explore the function of the SOC experimentally, we first employed two ex vivo models: (i) application of different loads to to bones of similar size but with (mouse) or without (rat) a SOC and (ii) application of identical pressure (load per area) to rat bones at different stages of development (before, during and after formation of the SOC).

We first compared similar rodent tibiae, one with and one without an SOC. Analysis of development pattern of mouse and rat bones revealed that the tibiae from 10-day-old rats and 30-day-old mice are comparable in size and shape (Figure 5-figure supplement 3A), as well as in the mechanical properties of the cartilage (Figure 5-figure supplement 3B). However, the rat tibia has not yet developed a SOC (referred to here as the SOC- model), whereas the murine tibia does have a well-developed SOC at this age (the SOC+ model) (Figure 1-figure supplement 1A-B). We applied vertical or angled loads, which represent the loading conditions at rest or during locomotion, respectively, with different forces to the SOC- and SOC+ models and first analyzed their physical properties. Consistent with the mathematical prediction, the SOC enhanced the stiffness and elasticity of these bones in connection with both vertical and angled loads (Figure 5 -figure supplement $3 \mathrm{C}-\mathrm{J}$ ). Although confounding variables cannot be definitively excluded, the agreement between the mathematical prediction and the physical tests further support our model that SOC provides additional stiffness, while simultaneously reducing octahedral shear stress within the epiphyseal growth plate.

To address the protective effect of SOC on epiphyseal chondrocytes, we placed bones exposed previously to different loads into in vitro culture conditions for 48 hours to allow load-induced cellular responses to manifest, using previously optimized culture conditions (30). We then assessed the survival status of the epiphyseal chondrocytes using exposure to propidium iodide (PI) during the end of the culture period (i.e., a part of live/death assay to visualize dead cells). These experiments revealed that chondrocytes appeared to be highly sensitive to load, with $40 \%$ dying upon application of a $1 \mathrm{~N}$-load in the SOC-model; whereas the presence of SOC allowed these cells to withstand a load one order of magnitude higher (Figure 5A-B and Figure 5-figure supplement 4). The unfavorable effect of loading on epiphyseal chondrocytes in both the SOC- and SOC+ models was confirmed by the decreased expression of several typical chondrocyte markers, such as Indian hedgehog (Ihh), parathyroid hormone-related peptide (PTHrP) (Figure 5-figure supplement 3K-L) and collagen type X (Col X) (Figure 5-figure supplement $3 \mathrm{~N}-\mathrm{O}$ ).

To distinguish between mechanical damage and stress-induced activation of self-elimination apoptotic program, we performed the TUNEL assay on the loaded bones to detect fragmented DNA, a hallmark of apoptotic nuclei. Epiphyseal chondrocytes in the SOC+ model become apoptotic in response to much higher loads as compared to the SOC- model (Figure 5C-E, see also Figure 5-figure supplement 3M for baseline control). In addition, much higher loads were required for SOC+ model to activate caspase-3, a protease that executes apoptosis (Figure 5F). To further explore the underlying mechanism, we checked the activity of the signaling pathway involving the Yes-associated protein 1/Tafazzin (YAP/TAZ), which is well-known to be involved in mechano-sensing (31) and can promote apoptosis via the tumor suppressor protein, p73 (32). Both the levels and nuclear translocation of the YAP (Figure 5- 
figure supplement 5A and Figure 9-figure supplement 6K-L) and p73 proteins (Figure 5-figure supplement $5 \mathrm{~B}$ ) were enhanced by loading, with their overlapping distribution pattern further confirming the activation of this signaling pathway (Figure 5-figure supplement 5C). Thus, caspase-dependent apoptosis, likely via the YAP-p73 pathway, appears to be triggered in epiphyseal chondrocytes by mechanical loads with a SOC providing substantial protection.

We also noticed that cell death was most extensive in the hypertrophic zone (Figure 6A-D), where the levels of active caspase-3, YAP and p73 were also increased to a greater extent in the hypertrophic zone than in the resting/proliferative zone (Figure 6E-H and Figure 6-figure supplement 7A-D). In SOC- bones hypertrophic chondrocytes died in response to a load as low as $0.2 \mathrm{~N}$ (Figure 6I), whereas in the SOC+ bones this phenomenon occurred only with loads of 3-5N (Figure 6A-D). Within this loading range, no effect on chondrocyte proliferation was observed (Figure 6-figure supplement 7E-F), suggesting that loading affects particularly the hypertrophic chondrocytes.

Despite agreement with the mathematical predictions, confounding variables cannot be definitively excluded in this SOC \pm model. To further strengthen this line of experimental evidences, we compared the effect of the same pressure (load per cross-sectional area) on rat tibiae before, during and after formation of the SOC. The tibia was isolated from rats of the same litter at 5, 8, 12, 15 and 22 days of age and then measured in order to apply a load (0.2$0.65 \mathrm{~N}$ ) that generated a pressure of of $0.023 \mathrm{MPa}$ per cross-sectional area. We found that a pressure identical to that which led to the death of chondrocytes prior to formation of the SOC did not affect the survival of these cells after this formation (Figure 7A-D). In addition, hypertrophic chondrocytes proved to be particularly vulnerable to this effect of pressure (Figure $6 \mathrm{E})$. The percentage of the hypertrophic chondrocytes that died and size of the SOC were negatively correlated (Figure 7F-G).

Thus, both these ex vivo models suggest that the SOC protects the growth plate chondrocytes from mechanical stress, the effect particularly pronounced in hypertrophic cell population, which renders these cells able to withstand approximately 25 -fold greater load when an SOC is present (comparing $0.2 \mathrm{~N}$ to $5 \mathrm{~N}$ ). In combination with mathematical modelling (compare the two left columns in Figure 1F), these findings indicate that the SOC protects chondrocytes and, especially, hypertrophic chondrocytes from the deleterious effect of extensive mechanical stresss.

Functional experiments with physiological systems: the SOC protects epiphyseal chondrocytes

This highly effective protection by SOC observed in the ex vivo models prompted us to modulate the size and maturity of the SOC in a series of in vivo experiments to compare the survival status of the epiphyseal chondrocytes. We recently reported that an inhibitor of vascular endothelial growth factor (VEGF) receptor, axitinib (33), can delay development of the SOC within a narrow developmental window, i.e., when axitinib is administrated at P18, but not $\mathrm{P} 21$ as analyzed in transgenic mice (34). Here, similar inhibition on the size of SOC was observed in wild-type mice (Figure 8A-B). The mice in which development of the SOC was delayed showed significantly higher levels of apoptosis in the hypertrophic zone than corresponding controls (Figure 8C-D). Axitinib treatment till the same final time point (P27) or for the same duration (P21-P30) was employed as controls in these experiments (Figure 8A, C). No significant effect of axitinib on mechanical properties of epiphyseal cartilage was observed as assessed by nanoindentation $(8.1 \pm 1.7 \mathrm{kPa}$ (mean $\pm \mathrm{SE}$, $\mathrm{n}=6$, control animals) and $10.5 \pm 1.9 \mathrm{kPa}(\mathrm{n}=7$, axitinib-treated animals $), \mathrm{p}=0.37$, unpaired $\mathrm{t}$ - test; 
mice were treated with vehicle or axitinib from P21 till P30 and live tissue sections were analyzed).

In a second model, we employed mice with limb-specific activation of the stimulatory G-protein $\alpha$-subunit (Gnas), which exhibit delayed SOC formation (PrxCre:Gnas ${ }^{R 21 H}$ ) (35). To block immediate elimination of dead hypertrophic chondrocytes by ingrowing blood vessels, we inhibited angiogenesis in these mice and corresponding controls by injecting axitinib at a time-point when it does not interfere with formation of the SOC (P21P30, Figure 8A), but delays elimination of hypertrophic chondrocytes (34). Analysis of these mice confirmed reduced SOC size in mutant mice and revealed elevated number of dead hypertrophic chondrocytes in these mice (Figure 8E-F). It must be emphasized that in both these pharmacological and genetic models, mice actively run and thereby expose their growth plates to the weight-associated loads, but have decreased body mass (for axitinib-treated mice, $56.6 \pm 11.2 \%$ of the DMSO-treated control and for the Prx-Cre:Gnas ${ }^{R 21 H}$ mice, $80 \pm 6.9 \%$ of the corresponding controls). Thus, the increase in cell death observed may be an underestimation of what might occur in bones with an underdeveloped SOC under normal weight-bearing conditions.

Summarizing, both pharmacological and genetic manipulation of the SOC confirmed its protective effect on hypertrophic chondrocytes, which is in agreement with the results obtained from ex vivo models and mathematical predictions.

Mechanical properties of hypertrophic chondrocytes make them vulnerable to the mechanical stress

In another mouse model examined, where formation of the SOC is delayed substantially by chondrocyte-specific ablation of the salt-induced kinase 3 (Sik3) (Col2al-Cre:Sik $3^{F / F}$ mice) (36), we noticed increased death of the hypertrophic chondrocytes not throughout the hypertrophic zone, but mostly at the sides of the growth plate (Figure 9A-B). In this strain hypertrophy is slightly delayed and we thought this observation might reflect a phenomenon overlooked in our previous experiments, i.e., higher load at the edges or its different distribution during locomotion. To explore this further, we applied loading to our SOC \pm model at an angle from lateral side and examined distribution of the cell death along the medial-lateral axis within the growth plate (Figure 9C). We noticed that chondrocytes on the side opposite to the loading underwent more extensive cell death (Figure 9D-E). This uneven distribution of cell death was also reflected in the higher number of TUNEL-positive cells and levels of YAP at the side opposite to the one where angled loading was applied (Figure 9-figure supplement 6C, E, G, I). Vertical loading did not affect the medial-lateral distribution of cell death, thereby serving as a control (Figure 9-figure supplement 6A, B, D, F, H, J).

This medial-lateral distribution of the cell death does not match the distribution of the octahedral stress, since upon angled loading this stress is low at the sides (Figure 1F). However, the distribution of the highest compressive principal stress and hydrostatic stress upon angled loading (Figure 1-figure supplement 2, see also Figure 9F and Figure 9-figure supplement $6 \mathrm{M}$ for physiological loading range) matches well with the observed distribution of cell death along the medial-lateral axis. Hydrostatic stress can be excluded, since it increases upon vertical loading in the central part and at edges, and therefore does not match distribution of cell death upon vertical load (Figure 1-figure supplement 2, Figure 9-figure supplement $6 \mathrm{M})$. Thus, we concluded that directional compressive (principle) stress appears to be the one that overlaps well with the spatial distribution of cell death under various loadings, and is likely, together with octahedral stress, harmful for hypertrophic chondrocytes. Vulnerability to compressive stress suggests that hypertrophic chondrocytes have a very low Young's modulus 
(i.e., the ratio of stress to strain). To check this directly, we utilized atomic force microscopy (AFM) to measure cell stiffness in live tissue sections as described (37). According to the mathematical prediction, we found that hypertrophic chondrocytes were only approximately $25 \%$ as stiff as the columnar chondrocytes from which they are derived (Figure 9G-H).

Therefore, it seems plausible that this relatively low stiffness of hypertrophic chondrocytes, in combination with their large size, render them particularly vulnerable to mechanical loading.

\section{The involvement of mechanical versus genetic factors in the development of the SOC}

As mentioned in the introduction, many investigators presently believe that formation of the SOC is stimulated by mechanical factors $(8,30,38-41)$. On the other hand, the observation that the thumb and fingers on the same wing of Chiroptera ossify at different times in utero (Figure 2) indicates that genetic factors may play a more important role in this formation. To explore this proposal, we examined the effects of unloading during limb development in rats and characterized ossification in a newt species during its terrestrial stage of growth.

On postnatal day 10, the hindlimbs of rats were unloaded either by resection of the sciatic nerve or fixation with kinesiology tape (which resembles fixation with a cast but is lighter and easier to change daily). Analysis at 23 days of age revealed that neither of these forms of unloading had affected the size of the SOC (Figure 10A-D, F-I), although in both cases bone mass was reduced dramatically (Figure $10 \mathrm{E}, \mathrm{J}$ ). The inability of the animals to use their nerve-resected leg was confirmed by testing footprint behavior (42) (Figure 10-figure supplement 8) and this leg was also insensitive to pricking with a needle (video-recordings made every 3-4 days are available upon request). Inability to use the taped leg was also documented by video-recording every 3-4 days (available upon request).

These experiments indicate that the development of the SOC is primarily under genetic control. To obtain further support for this conclusion, we analyzed the epiphysis in the salamander Notophthalmus viridescens, anamniotes that do not normally develop a SOC (Figure 1D). However, these animals can be raised under terrestrial conditions for several months. Therefore, we raised the newt Notophthalmus viridescens first under aquatic conditions, thereafter under terrestrial conditions (the eft stage, characterized by much slower growth than during the aquatic stages (43)) and then under aquatic conditions again. No SOC was formed during terrestrial growth (Figure 10-figure supplement 9A), although calcification of cartilage during this period could be detected by Von Kossa staining (Figure 10-figure supplement 9A, middle panel). The numbers of apoptotic (Figure 10-figure supplement 9B) and proliferating chondrocytes (Figure 10-figure supplement 9C) were similar under aquatic and terrestrial conditions.

Altogether, these observations indicate that development of the SOC is governed primarily by genetic mechanisms designed to meet future mechanical demands, which aligns well with the evolutionary and zoological observations.

\section{Discussion}

We show here that the SOC protects hypertrophic chondrocytes from mechanical stress and likely appeared in evolution in association with this function. We found that hypertrophic chondrocytes are characterized by a very low mechanical stiffness and high vulnerability to loading. It is likely that their low mechanical stiffness in combination with the large size makes them vulnerable to various mechanical stresses, including the octahedral and principle compressive stresses. These mechanical vulnerability may be intrinsic to the process of 
chondrocyte hypertrophy per se, which is characterized by enormous and rapid increase in the cellular volume, i.e., 20 folds within 12 hours (44). At the same time, chondrocyte hypertrophy is a key part of the process of endochondral bone formation, not only contributing $59 \%$ of longitudinal growth $(73 \%$ if the corresponding extra-cellular matrix is taken into account $(45$, 46)), but also coupling cartilage resorption to the formation of the underlying bone tissue (e.g., via secretion of Ihh and VEGF, as well as matrix calcification) (47). The growth of long bones via hypertrophic intermediates (i.e. endochondral bone formation) has been evolutionarily conserved for at least $400 \pm 20$ Mya $(3,4)$ and it seems plausible that early in evolution, when growth occurred in aquatic environment, no specific mechanical demands were posed on the hypertrophic chondrocytes due to compensation of body mass/ground reaction force by buoyant force. However, transition to fully terrestrial environment, unequivocally coupled with the loss of the buoyant force, places new mechanical demands on the growing skeleton, such as a need to withstand the body mass, and required additional adaptations of the skeleton.

The observation that longitudinal growth is sensitive to mechanical loads was documented almost two centuries ago and formulated during the period of 1862-1897 as the Hueter-Volkmann law, which states that "increased mechanical compression reduces longitudinal bone growth while reduced mechanical loading increases it" (48). This law was confirmed by numerous subsequent studies. For example, Matsuda et al. (49) revealed that the growth of the tarsometatarsus of roosters was delayed when exposed to a strenuous exercise. This observation was further extended by Reich et al. (50), who showed that chicks freely running with backpacks loaded with $10 \%$ of their body mass experience growth retardation associated with increased resorption of the epiphyseal cartilage. Kiiskinen (51) showed that the longitudinal growth of the femur of young mice was retarded after several weeks of intensive training. Our data suggest that such a sensitivity of the growing bones to excessive load is due to the mechanical properties of hypertrophic chondrocytes and their vulnerability to mechanical stresses. It is likely that the cell death observed in our ex vivo and in vivo experiments is rather a far extreme manifestation of the cellular stress experienced by hypertrophic chondrocytes. The lower levels of cellular stress might have lesser, but yet negative impact on the hypertrophy process, such as decreased production of extracellular matrix and signaling proteins, including the observed reduction in Ihh, PTHrP and ColX expression. Supporting, decreased expression of ColX was observed in the chicks with backpacks (50).

Locomotion is thought to impose the most frequent and severe loads on limb bones in terrestrial tetrapods $(52,53)$. In children, normal gait pattern generates peak loads of 4-6 folds of the body weight on the femur $(54,55)$. Such loads would be expected being even higher during intensive physical activity, like sprinting or jumping $(54,56,57)$. Thus, the load of $0.2 \mathrm{~N}$ used in the present study is within the physiological range for animals with a 20-gram body weight and would impair hypertrophic chondrocytes if they are not protected. Therefore, it is plausible to assume that if mice would not develop the SOC, hypertrophy of their chondrocytes would be negatively impacted during locomotion, which is in line with our pharmacological and genetic manipulations where mice with under-developed SOC display increased death of hypertrophic chondrocytes. At the same time, our data show that the SOC provides a very effective protection, increasing withstanding loads up to 25 -folds and likely allowing hypertrophic chondrocytes to bear the corresponding body weight under various locomotion modalities, including sprinting and jumping.

However, in absolute numbers the loads in elephants and mice look far beyond any compensation achievable by the SOC. How does it work? Considering species with various body masses along the phylogenetic tree, it was first noted by Galileo (58) that the skeletal elements become more robust relative to the body mass (BM) as the latter increases. The 
relation of the skeletal elements to BM is allometric (i.e. adaptive) and is generally described by the power law equation $y=k x^{a}$, where $k$ represents coefficient and $a$ represents scaling factor. The highest correlation has been shown between stylopodial (femur or humerus) diameter (D) and body mass (59). However, the exact scaling factor varies among studies, depending on the type of species and taxa utilized, weight-range selection, number of outliers, statistical strategy, sample size, etc. For example, for ungulates this relationship is calculated as $\mathrm{D}=\mathrm{BM}^{0.366}(60)$, whereas for insectivores+rodents it is $\mathrm{D}=\mathrm{BM}^{0.3944}(61)$. The most comprehensive analysis of quadrupedal terrestrial tetrapods (including both mammals and reptiles) resulted in the equation $\mathrm{D}=1.26^{*}(\mathrm{BM})^{0.364}(59)$. This allometric increase in bone diameter substantially reduces the pressure (load per area) on the growth plate with increased bone mass. For example, due to this allometry the increase in body size by 1000 folds elevates the pressure on the growth plate by 4.3-6.6 folds (depending on which scaling factor from above is employed). Thus, allometric scaling of the skeleton compensates for most of the mechanical stress associated with increased body mass in terrestrial tetrapods. However, this compensation is not complete and pressure on the growth plate gradually increases for heavier animals. Why this compensation is not complete is currently unclear but may be coupled to high energy demands or unbearable skeletal mass required for compensation exclusively via skeletal allometry. Indeed, simple calculations show that complete pressure compensation on the skeleton can be achieved with the scaling factor 0.5 (i.e., $\mathrm{D}=\mathrm{BM}^{0.5}$ ), which would result in 3.4 folds increase in diameter of every weightbearing skeletal structure of a $10 \mathrm{~kg}$ animal, such as a dog. Various alternative adaptations are employed by nature to allow the skeleton to withstand the increasing body mass and preserve the bone safety factor (the ratio of failure load to functional load), which is surprisingly stable from mouse to elephant and ranging from two to four (52). The most significant adaptations include musculoskeletal composition, postures and gait patterns $(52,62)$. These adaptations, thought to evolve for preservation of the skeletal safety factor, may unequivocally provide additional protection to the growth plate structure.

These various adaptations need to be considered from the evolutionary perspective, where various traits can evolve independently, develop in parallel or converge. With few sub-specialized exceptions, the entire Mammalia class can be characterized by the development of the SOC. In contrast, within Sauropsida clade, which is subdivided into two clades, Lepidosauria and Archosauria, limbed lepidosaurs (lizards and Sphenodon) exhibit an SOC, whereas Archosauria (crocodilians, chelonians, birds and dinosaurs) exhibit ossified protrusions of bone marrow into epiphyseal cartilage $(6,7,12,13)$ (Figure 1E).. These protrusions are well-developed with calcified or ossified walls and penetrating deep into the epiphyseal cartilage, sequestering hypertrophic chondrocytes in between them in non-avian dinosaurs and birds, but very rudimental in chelonians and crocodilians $(6,7,12,13)$. According to our mathematical modeling, these extended protrusions are more efficient in protecting the zone of hypertrophic chondrocytes, whereas the SOC also reduces deflection of the joint during locomotion (Figure $1 \mathrm{~F}$ ), which would facilitate a more efficient transmission of the ground reaction force vector toward the body movement. Whether this difference has an evolutionary advantage remains to be explored, but the observation that extant birds develop SOCs only in their weight-bearing long bones (63) might suggest that improving of the mechanical properties of the cartilaginous joint has an additional evolutionary advantage. Thus, it is plausible that the appearance of the SOC in weight-bearing long bones of birds represents an evolutionary convergence. At the same time, the appearance of the SOC in Mammalia and Lepidosauria and ossified protrusions in Archosauria can be considered as alternative parallel traits. Interestingly, recent genome sequencing of tuatara, the most ancient extant creature of 
Lepidosauria, revealed sparkling similarities in genome architecture with mammalian lineages (64).

Of course, evolution of skeletal traits should not be considered alone, but in a connection with other related traits, such as postures and gaits. In this context, Mammalia can be characterized by an erect posture with (nearly) parasagittal limb kinematics in which the limbs support the entire weight of the trunk (65-67). Same as mammals, birds and dinosaurs are characterized by an erect posture $(68,69)$ whereas limbed lepidosaurs, crocodilians and chelonians have a sprawling posture with laterally projected limbs (70). In such a configuration, the weight of the body is essentially distributed as shear stress in the midshaft of the stylopod, thereby loading the bone in torsion $(53,67,71,72)$. The hypertrophic chondrocytes are therefore not highly stressed in compression. Contrary to chelonians, some limbed lepidosaurs and crocodilians are capable of semi-erect posture $(73,74)$. This is an intermediate configuration between the sprawling and erect posture (70). In that position, during the phases of fast locomotion, limb-bone strain can significantly increase in axial compression and bending $(67,75-77)$. Crocodilians partition their habitat (to maximize feeding and/or minimize interference and predation), the juveniles are often restricted to shallow aquatic margins (7880). They barely go out of the water and only occasionally use a semi-erect posture. For these reasons, it is likely that very little compressive stress is applied to their limb-bone growth plates at the juvenile stage. This may be the reason why crocodilians develop no SOC at their limbbone extremities (6). On the contrary, all lizard limb-bones have SOCs $(6,7,81,82)$ or calcified secondary center (SC) (in sphenodontids, Figure 1D) (83). Lizards are terrestrial at the juvenile stage. In this exposed environment, they need to sprint fast and jump to avoid predation and hunt (84-87). Many of them use a semi-erect or bipedal posture for this $(70,73,74)$. Thus, evolvement of the SOC may provide an advantage in terms of protection of the hypertrophic chondrocytes, simultaneously with improved firmness of the joint structure during juvenile stage. Tortoises also develop exclusively on land. However, they only display this lateral limb projection typical of sprawling posture, which induces a shear stress on the femoral midshaft (88). No compression has been reported so far in limb bones of tortoises. In addition, they move in such a slow manner $(53,88-90)$ that the threshold of strain-related stimuli is too low (53) to constrain the microanatomy of limb bones (91) and stress their growth plates. This probably explains why SOCs are not present in terrestrial tortoise limb bones.

These evolutionary considerations lead us to propose that the development of SOCs (in mammals and Lepidosauria) or extended ossified protrusions (Archosauria) can be considered as parallel evolutionary traits evolved together with various postures for adaptation to the weight-bearing demands of terrestrial environments, including the mechanical stress placed on the epiphyseal cartilage in juveniles. At the same time, evolutionary transition from water to land was associated with numerous other changes in the endocrine, respiratory, secretory and other systems, most of which exert or may exert various effects on the skeleton. For example, the physiological functions of calcium influence the regulation of numerous types of bone cells. Similarly, the translocation of hematopoiesis from the kidney in fish to the bone marrow in terrestrial animals also affects bone physiology. Thus, mechanical adaptation may not be the only explanation for development of the SOC.

From an evolutionary perspective, adaptive development of the SOC suggests an underlying genetic mechanism(s), as opposed to the widely held view that mechanical stress causes this development $(8,30,38-41)$. Indeed, in many instances the SOC is formed during development prior to the application of mechanical forces. One well-known example of this is provided by newborn marsupials (e.g., the kangaroo), which are quite immature when born and need to use their forelimbs (which are much more ossified at this point than their hindlimbs) to 
climb into the pouch (92). Another example involves ungulates, who must be able to walk and run within a few hours after birth. Accordingly, in calves the SOC develops well before birth $(93,94)$. The heterochrony in the development of the SOC in different bones of Jerboa and Chiroptera observed here is in line with these observations. Furthermore, such temporal variation even before birth and within the same limb of Chiroptera prepares adequately for future mechanical demands (see further above). Furthermore, early mechanical unloading of the hindlimbs of rats had no effect on the development of the SOC and raising Notophthalmus viridescens, which do not naturally develop an SOC, under terrestrial weight-bearing environment did not promote formation of this structure (95). All these findings indicate strongly that development of the SOC is genetically programed.

The nature of this genetic programming remains to be elucidated. Interestingly, it was reported recently that a single nucleotide neomorphic mutation in miRNA140 delays appearance of the SOC substantially, without dramatically influencing chondrocyte hypertrophy (96), suggesting that the underlying genetic program can be fine-tuned relatively easy. Among potential endocrine factors, thyroid hormone $(\mathrm{TH})$, which regulates skeletal development in all vertebrates (97), promotes development of the SOC. In TSHR-deficient mice formation of the SOC is delayed substantially and this effect can be reversed by T3/T4(98). Moreover, inactivation of thyroid hormone receptor - alpha (THR1a) in chondrocytes impairs their hypertrophy, formation of the SOC and overall bone growth (99). At the same time, the SOC develops at different time-points in different bones suggesting the involvement of local mechanism(s). Indeed, sophisticated pre-receptor control of the activity/availability of TH (e.g., through tissue-specific expression of deiodinase enzymes and transporters) and of their receptors (e.g., through issue-specific expression of these receptors and their co-activators and co-repressors) allows tight regulation of the timing and location of responsiveness to this hormone (100). Since TH influences the expression of approximately 400 early-response genes in the growth plate (99) and roughly the same number of genes during metamorphosis in amphibia (101), it is difficult at present to even speculate about which specific genetic program triggered by $\mathrm{TH}$ might be involved in regulating development of the SOC.

In conclusion, various independent approaches employed in this study (i.e., evolutionary analysis, mathematical modeling, biophysical tests, comparative zoology, biological experiments) all suggest that one evolutionary reason for the appearance of a spatially separated growth plate is to shield the hypertrophic chondrocytes from the mechanical stress associated with weight-bearing and, accordingly, preserve the mechanism of endochondral bone formation in terrestrial animals. Conceptual understanding of the underlying evolutionary, mechanical and biological reasons behind spatial separation of articular cartilage and the growth plate will provide deeper understanding of skeletal biology and evolution. This, for example, can improve the design of sporting activities for children or influence surgical interventions related to epiphyseal fractures.

\section{Materials and Methods}

Key Resources Table

\begin{tabular}{|l|l|l|l|l|}
\hline $\begin{array}{l}\text { Reagent type } \\
\text { (species) or } \\
\text { resource }\end{array}$ & Designation & Source or reference & Identifiers & $\begin{array}{l}\text { Additional } \\
\text { information }\end{array}$ \\
\hline
\end{tabular}




\begin{tabular}{|c|c|c|c|c|}
\hline $\begin{array}{l}\text { C57BL/6 } \\
\text { (mouse strain) }\end{array}$ & & $\begin{array}{l}\text { Charles River } \\
\text { Laboratories }\end{array}$ & & \\
\hline $\begin{array}{l}\text { Sprague } \\
\text { Dawley (rat } \\
\text { strain) }\end{array}$ & & Janvier Labs & & \\
\hline $\begin{array}{l}\text { Col2al- } \\
\text { Cre:Sik3 } 3^{F / F} \\
\text { (mouse strain) }\end{array}$ & & $\begin{array}{l}\text { Dr. Henry } \\
\text { Kronenberg, } \\
\text { Massachusetts } \\
\text { General Hospital }\end{array}$ & & \\
\hline $\begin{array}{l}S i k 3^{F / F} \text { (mouse } \\
\text { strain) }\end{array}$ & & EUCOMM & & \\
\hline $\begin{array}{l}\text { Col2al-Cre } \\
\text { (mouse strain) }\end{array}$ & & $\begin{array}{l}\text { Richard R. } \\
\text { Behringer, } \\
\text { University of } \\
\text { Texas MD } \\
\text { Anderson Cancer } \\
\text { Center }\end{array}$ & & \\
\hline $\begin{array}{l}\text { Prx- } \\
\text { Cre:Gnas } \\
\text { (mouse strain) }\end{array}$ & & $\begin{array}{l}\text { Dr. Murat } \\
\text { Bastepe, } \\
\text { Massachusetts } \\
\text { General Hospital }\end{array}$ & & \\
\hline antibody & $\begin{array}{l}\text { cleaved } \\
\text { caspase } 3 \\
\text { (rabbit } \\
\text { polyclonal) }\end{array}$ & Cell Signaling & \#9661 & $1: 500$ \\
\hline antibody & $\begin{array}{l}\text { YAP (mouse } \\
\text { monoclonal) }\end{array}$ & Santa Cruz & $\begin{array}{l}\text { sc- } \\
101199\end{array}$ & $1: 50$ \\
\hline antibody & $\begin{array}{l}\text { p73 (rabbit } \\
\text { polyclonal) }\end{array}$ & Abcam & ab137797 & $1: 100$ \\
\hline antibody & $\begin{array}{l}\text { ki67 (rabbit } \\
\text { monoclonal) }\end{array}$ & Invitrogen & $\begin{array}{l}\text { MA5- } \\
14520\end{array}$ & $1: 20$ \\
\hline antibody & $\begin{array}{l}\text { MCM2 } \\
\text { (rabbit } \\
\text { polyclonal) }\end{array}$ & Abcam & ab4461 & $1: 250$ \\
\hline
\end{tabular}

Ethics

617 Except for those specified below, all animal experiments were pre-approved by the Ethical 618 Committee on Animal Experiments (N5/16, N187/15, 9091-2018, Stockholm North 619 Committee/ Norra Djurförsöksetiska Nämnden), the Institutional Animal Care and Use 
620 Committee of the Massachusetts General Hospital (Protocols \#: 2005N000094 and 621 2004N000176) or the University of California San Diego (D16-00020) and conducted in 622 accordance with the provisions and guidelines for animal experimentation formulated by the 623 Swedish Animal Agency. Animal experiments involving limb unloading, AFM and 624 nanoindentation were pre-approved by the Ethics Committee of the Sechenov First State Moscow Medical University (No. 07-17 from 13.09.2017, Moscow, Russia).

\section{Synchrotron scanning and 3D modeling}

All these scans were performed at the beamline ID19 (European Synchrotron Radiation Facility, France) with a current of $200 \mathrm{~mA}$ or 16 bunch. Scanning and 3D-modeling of the humerus of Eusthenopteron were performed as described by (3). The data were reconstructed using a single distance phase retrieval approach(102) based on a modified version of the algorithm of (103), applying an unsharp mask to the radiographs after the phase retrieval to enhance the trabecular mesh. The humerus of Seymouria was imaged with a voxel size of 3.48 $\mu \mathrm{m}$ using an optical system associated to a $47 \mu \mathrm{m}$-thick GGG (i.e., a gadolinium gallium garnet crystal) scintillator and a FreLON 2k14 CCD detector (104). The propagation distance was of $700 \mathrm{~mm}$. The gap of the W150m wiggler was opened to $37 \mathrm{~mm}$. The sample was imaged at 80 $\mathrm{keV}$ using a beam filtered with $0.25 \mathrm{~mm}$ of tungsten and $2 \mathrm{~mm}$ of aluminium. In half-acquisition conditions, 4998 projections were produced over $360^{\circ}$ with a time of exposure of $0.15 \mathrm{~s}$. Binned images (final $6.96 \mu \mathrm{m}$ voxel size) were reconstructed, segmented and 3D-modeled in the same manner as Eusthenopteron (3). The scan of the humerus of Sciurus vulgaris was made with the same voxel size $(3.48 \mu \mathrm{m})$ as for the humerus of Seymouria using the same optics (FreLON $2 \mathrm{k} 14$ CCD camera and a $47 \mu \mathrm{m}$-thick GGG scintillator). The sample was imaged with a propagation distance of $500 \mathrm{~mm}$. The beam was filtered with $0.14 \mathrm{~mm}$ of tungsten, $0.14 \mathrm{~mm}$ of copper and $2.8 \mathrm{~mm}$ of aluminium. The gap of the $\mathrm{W} 150 \mathrm{~m}$ wiggler was opened to $50 \mathrm{~mm}$, which resulted in an energy of $65.8 \mathrm{keV}$. In half-acquisition conditions, 4998 projections were produced over $360^{\circ}$ with a time of exposure of $0.15 \mathrm{~s}$.

\section{MicroCT scan of Pipistrellus pipistrellus s.l.}

The 1-3-day-old bat was scanned in Vienna with a SkyScan 1174 as previously described (105).

\section{Mice and rats}

30-day-old C57BL/6 mice (body weight 14.59 $\pm 0.72 \mathrm{~g}$ ) and 10-day-old Sprague Dawley rats (body weight $20 \pm 0.49 \mathrm{~g}$ ) were purchased from Charles River Laboratories and Janvier Labs (Europe). C57BL/6 mice used for the axitinib experiments were purchased from Charles River Laboratories. Col2al-Cre:Sik3 ${ }^{F / F}$ mice were obtained from Dr. Henry Kronenberg, Massachusetts General Hospital. The $S i k 3^{F / F}$ mice were originally purchased from EUCOMM and the Col2al-Cre mice were obtained from Richard R. Behringer, University of Texas MD Anderson Cancer Center (106). Prx-Cre:Gnas ${ }^{R 201 H}$ mice were generated by Dr. Murat Bastepe, Massachusetts General Hospital and were described previously (35).

\section{Dissection, loading and culturing of the tibia and femur}

From both hind legs of 30-day-old mice and 10-day-old rats, the tibia and femur bones were dissected out aseptically in DMEM/F12 medium supplemented with $50 \mu \mathrm{g} / \mathrm{ml}$ gentamycin (dissection medium) on ice, with removal of as much surrounding tissue as possible. The height and diameters of the top and side surfaces of the epiphysis were measured with a digital caliper.

667 All comparisons involved the bones from the same animal. 
668 Still in dissection medium on ice, the bones were subjected to pressure with the Instron ElectroPuls E1000 Test Instrument. Load was applied at a speed of $0.1 \mathrm{~N}$ per second and load/digital position curves recorded in the Bluehill 3 software. Control bones went through the same procedures, but without loading. For culturing, bones were first washed twice in dissection medium before being placed into DMEM/F12 medium supplemented with $1 \mathrm{mM} \beta$-glycerophosphate, $0.2 \%$ bovine serum albumin, $50 \mu \mathrm{g} / \mathrm{ml}$ ascorbic acid and $50 \mu \mathrm{g} / \mathrm{ml}$ gentamycin for 48 hours as described previously (28). Propidium iodide (stock solution at $1 \mathrm{mg} / \mathrm{ml}$, Sigma) was added to the culture medium at a ratio of 1:50 30 minutes before termination of culturing. The tibia and femur were then fixed in $4 \%$ PFA overnight, decalcified, cleared in $30 \%$ sucrose overnight (at $4^{\circ} \mathrm{C}$ ) and finally embedded in optimal cutting temperature (OCT) compound for frozen sectioning. When applying the load, the medial tibia plateau flanked by the tibia bone grooves is always facing one direction and the angled pressure was applied always to one side of the plateau (on top of one of the grooves). When embedding in OCT for sectioning, the bone is always placed into the grid with the medial tibia plateau facing upward and sectioned frontally to ensure the pressure direction during imaging.

\section{Calculation of mechanical and material properties}

Determination of the position of the bone relative to the position of its fixture yielded the deformation value. Stiffness was defined as the slope of the initial linear portion of the loaddeformation curve. Stress was calculated by distributing the load equally over the top (vertical load) or side surface (angular load) of the epiphysis, assuming that both of these surfaces were oval. Strain was calculated by distributing the bone deformation equally along the height of the epiphysis. The initial linear portion of the stress-strain curve up to $0.2 \%$ offset was plotted together with the stress-strain curve itself in the same graph and the intersection of the line and curve defined as the $0.2 \%$ offset yield strength.

\section{Axitinib Injection}

Axitinib (Sigma) was dissolved in DMSO and injected intraperitoneally at a dose of $0.25 \mathrm{mg}$ per animal per day for the C57BL/6 mice for 8 (P21 to P27) or 10 days (P18 to P27 or P21 to P30) Control group of the C57BL/6 mice receive the same volume of DMSO injection each day. Transgenic Prx-Cre:Gnas ${ }^{R 201 H}$ mice and corresponding Gnas ${ }^{R 201 H}$ controls were injected daily $0.017 \mathrm{mg}$ per gram of body weight between postnatal 21 and 29 days of age to block elimination of dead hypertrophic chondrocytes by ingrowing blood vessels. Animals were sacrificed one day after the last axitinib injection.

\section{TUNEL staining}

$30-\mu \mathrm{m}$ thick sections of cultured tibia bones were treated with proteinase $\mathrm{K}$ (Ambion) at 10 $\mu \mathrm{g} / \mathrm{ml}$ for 40 minutes at $37^{\circ} \mathrm{C}$ before applying the TUNEL reaction mix (Roche Inc.) for 90 minutes. The cells were then counter-stained with DAPI for 30 minutes.

\section{Quantification of positive cells (PI+, TUNEL+, YAP+ etc)}

For each data point of the PI+ and TUNEL+ cell number quantification, at least three sections per animal and at least three animals were analyzed. Double blind counting was performed for all quantification analysis, including the medio-lateral distribution of PI+, TUNEL+, Caspase$3+, \mathrm{p} 73+, \mathrm{YAP}+$ and double p73+YAP+ cells.

\section{Immunofluorescent staining}


$30-\mu \mathrm{m}$ sections of cultured tibia bones were blocked in $3 \%$ normal horse serum for one hour, followed by overnight incubation with primary antibodies against cleaved caspase 3 (1:500) (Cell Signaling), YAP (1:50) (Santa Cruz), p73 (1:100) (Abcam), Ki67 (1:20) (Invitrogen) or MCM2 (1:250) (Abcam) at $4^{\circ} \mathrm{C}$. Secondary antibodies tagged with fluorophore were then added for one hour followed by DAPI counter-staining for 30 minutes.

\section{In situ hybridization}

In situ hybridization was performed on 30- $\mu \mathrm{m}$ sections as described previously(107) utilizing a probe against ColXa1 (a gift from Prof. Bjorn Olsen, Harvard Medical School) labelled with digoxigenin (DIG) in accordance with the manufacturer's instructions (Roche Inc.). Samples were then treated with either DIG antibody Fab fragments (Sigma) and NTM (Sigma) for colorimetric visualization or DIG antibody HRP (Sigma) and TSA kit (Perkin Elmer) for fluorescent visualization.

\section{Atomic Force Microscopy}

$731 \quad 120-150-\mu \mathrm{m}$ thick sections of live rat tibia bones were obtained by vibratome (Thermo Fisher Scientific) and mounted onto glass discs using silicone grease from the Bruker fluid cell accessory kit (Bruker, USA). The force spectroscopy measurements were performed using a MultiMode 8 atomic force microscope with a Nanoscope V controller and E scanner (Bruker). The regions of cells of interest for the acquisition of force-distance curves were selected under the optical microscope in combination with the AFM instrument.

The force-distance curves were acquired employing CP-PNP-BSG colloidal probes (NanoandMore $\mathrm{GmbH}$, Germany) with a $5-\mu \mathrm{m}$ borosilicate glass microsphere attached to the $200-\mu \mathrm{m}$ cantilever. The spring constants of the cantilevers (measured by the thermal tune procedure) were $0.06-0.09 \mathrm{~N} / \mathrm{m}$.

741 All measurements were conducted at $25^{\circ} \mathrm{C}$ and all tissue was handled in DMEM/F12 HEPEScontaining medium on ice. At least 70 individual force-distance curves were acquired for each type of cell by ramping over the surface and totally 30 cells were measured from 3 different animals. These force-distance curves were processed with the NanoScope Analysis v.1.10 software (Bruker). Utilizing retract curves, the elastic modulus E was extracted from these force-distance curves by fitting in accordance with the Hertzian model of contact mechanics.

\section{Nanoindentation}

Local mechanical characteristics of 10-day-old rat and 30-day-old mice cartilage samples were measured with a Piuma Nanoindenter (Optics11, Netherlands) adapted for soft materials. Measurements were performed on 220-250- $\mu$ m sections of alive tissues (without fixation and freezing) obtained by vibratome (Thermo Fisher Scientific) at $2-4^{\circ} \mathrm{C}$. After the measurement, the sections were stained with PI (Sigma) and CalceinAM (Sigma) to confirm cell viability. Nanoindenter includes a controller, an optical fiber and a spherical tip for the forcedisplacement curves acquisition. The tip is attached to a flexible cantilever, the displacement of which after the contact with a surface is measured using an interferometer via an optical fiber.

758 To measure the Young's modulus the probe was immersed for $5 \mu \mathrm{m}$ into the sample at each

759 point of measurement. The Young's modulus for each point was computed according to the

760 Hertzian contact mechanics model for a spherical body indenting a flat surface, using the built-

761 in Piuma software.

762 For the study of 10-day-old rat and 30-day-old mice cartilage mechanical characteristics, we 763 used a cantilever with the spring constant of $4.14 \mathrm{~N} / \mathrm{m}$ and a tip with the $45.5 \mu \mathrm{m}$ radius of 
curvature. The measurements were conducted in Phosphate buffered saline (PBS) cooled to 2$4^{\circ} \mathrm{C}$ to maintain cell viability. The samples were immobilized with specially designed holder immersed in cold PBS. During the measurements the probe was always located inside the fluid medium at a sufficient depth, in order to avoid measurement errors due to adhesion forces at the air-water boundary. The area of the Young's modulus mapping for rat samples was $1350 \times 400 \mu \mathrm{m}$ with the step of $45 \mu \mathrm{m}$ by the $\mathrm{X}$-axis and $100 \mu \mathrm{m}$ by the $\mathrm{Y}$-axis. For mice the area of mapping was $585 \times 360 \mu \mathrm{m}$ with the step of $45 \mu \mathrm{m}$ by the $\mathrm{X}$ - and $\mathrm{Y}$-axes. Based on the results of the measurements, the effective Young's modulus was computed, its distribution over the surface was plotted and mean \pm standard deviation (SD) Young's modulus value calculated. For the experiment assessing the effect of axitinib on the mechanical properties of epiphyseal cartilage, between 14 and 96 measurements of cartilage stiffness with nano-indenter were performed for each animal and the median (due to not normal distribution of the measurements) was chosen as the closest "true" value representing cartilage stiffness for each mouse ( 6 controls and 7 treated mice). These values were employed to assess the effect of the treatment, their normal distribution verified by the Shapiro-Wilk test (passed; $\mathrm{P}=0.4051$ and $\mathrm{P}=0.3921$ for control and axitinib groups, respectively) and equality of distributions compared by unpaired $t-$ test with $\mathrm{n}=6$ for control and $\mathrm{n}=7$ for treated.

\section{Finite Element Analysis}

The effects of an SOC on the distribution of stress in the zones of bone growth were explored by numerical simulations. Following the reasoning of (14), a plane-strain 2D domain was considered, to simplify the complex geometry of the bone end, and thereby clarify fundamental aspects of the response. The model thus took five sub-domains into consideration. The geometry of this simulation domain was a slight modification of (14), with a total height of 66 $\mathrm{mm}$, radius at the top of $17 \mathrm{~mm}$, and bottom width of $25 \mathrm{~mm}$. The cortical bone was given a width of $2.5 \mathrm{~mm}$ (measured horizontally). The thickness normal to the $2 \mathrm{D}$ plane was by definition $1 \mathrm{~mm}$. Only the top part of the model is shown in results figures.

The main parameter in these simulations was the presence or absence of an SOC or other stiffer tissues, introduced by providing this domain with a stiffness at least equal to that of the cartilage domain. Non-linear strains were taken into consideration, but the materials in all subdomains were considered to be linearly elastic, as described by a Young's modulus $\mathrm{E}_{\mathrm{i}}$ and a Poisson ratio $v_{\mathrm{i}}$. We used the same material properties as (14), and in particular for cartilage $\mathrm{E}_{\mathrm{C}}=6 \mathrm{MPa}$, $v_{C}=0.47$. When included, the $S O C$ was defined in a subdomain with $E_{D}=500 \mathrm{MPa}, v_{D}=0.20$. Thus, the stiff tissue had the material properties of dense cancellous bone, i.e., approximately 80-fold stiffness compared to cartilage.

In all cases, the bottom edge of the complete model had zero displacement vertically, and the outside edges of the cortical bone zero displacement horizontally. Applied to different $45^{\circ}$ sectors of the semi-circular top, the pressure was modelled as maximal on the center of the given sector (300 $\mathrm{kPa}$ for physiological level, 3MPa for supra-physiological), falling quadratically and symmetrically to zero on the two neighboring sector borders. In light of the symmetry of the domain, only the top or right-hand side was loaded (except for a verification case to (14), not shown). The direction and integrated total force of loading were affected slightly by the finite deformation of the domain.

Different components or comparison values of stress (measured as the second Piola-Kirchhoff stress relative to the unstressed reference volume) were evaluated. Primary focus was on the hydrostatic stress, the lowest principal stress (always compressive), and the octahedral shear stress, providing different perspectives on the response to external loading. Since the situations considered were dominated by compressive stresses, the signs for the two first have been 
812 changed in figures. Stress values are plotted on the deformed shape of the domain, without 813 magnification of the deformation.

814 Numerical simulations of the response to loading were based on parameterized finite element 815 approximations, and performed in the Comsol Multiphysics software (version 5.2, Comsol AB, 816 Stockholm, Sweden.)

817 With similar loads, this model resulted in a stress distribution in and deformation of the cartilaginous epiphysis closely similar to those reported by (14). Model design for stem tetrapods and archosaurs were based on $(3,4)$ and $(10,11)$ respectively.

\section{Quantitative PCR}

822 Proximal end growth plate of mouse and rat tibia was dissected and homogenized in liquid nitrogen for subsequent RNA extraction by Trizol and RNA purification by the RNeasy kit (Qiagen). Only RNA samples with an A260/A280 value between 1.8 and 2.0 were used. Extracted RNA was reverse transcribed into cDNA using a kit from Takara. qPCR was performed using the SYBR Green I supermix (BioRad) and analyzed using the $\Delta \mathrm{Ct}$ method by normalizing with the house keeping gene GAPDH. Three growth plates from independent animals were analyzed for each condition.

ciatic nerve resection

831 Wistar-Kyoto rats at P10 were anesthetized with $20 \mathrm{mg} / \mathrm{kg}$ Zoletil® (Vibrac). On posterior side of the right leg, the skin and muscle were dissected, and the sciatic nerve was isolated. For experimental animals, a $5 \mathrm{~mm}$ nerve fragment was excised; for sham operated animals, the nerve was returned to the soft tissue without resection. The skin at the operation site was sutured and, after recovery from anesthesia, the pups were returned to their mother. The suture was treated with povidone-iodine for 7 days after surgery. Foot sensitivity was tested at P16 using a needle. Video recordings of gaits were carried out at P11, P14, P20 and P22. The animals were sacrificed at P23 for tissue collection.

\section{Leg immobilization with kinesiology tape}

841 The right legs of 10-day-old Wistar-Kyoto rats were immobilized using kinesiology tape (Bradex) and the left legs remain intact. Free living animals were used as the control group. The tape was wrapped in two layers to fix the knee and ankle joint. As the rats grew, the tape was changed every two days. Rats' gait pattern was observed daily, and video was recorded at P14, P20 and P22, to verify unloading of the taped leg. Rats were sacrificed at P23 for tissue collection.

\section{SOC size evaluation}

849 Knee samples were fixed in $3.7 \%$ formaldehyde for 1 day and decalcified in EDTA. $20 \mu \mathrm{m}$ 850 cryosections were stained with Safranin O and Fast Green. SOC size was estimated in the middle of the epiphysis, identifiable by the presence of a cruciate ligament. SOC area was measured and normalized to the total epiphysis area, including the growth plate with Image J.

Bone density evaluation

855 The primary spongiosa area (500 $\mu$ m under the growth plate) on hematoxylin and eosin stained sections was used to evaluate the bone density. Bone area that are stained with eosin was measured and normalized to the total primary spongiosa area with Image $\mathrm{J}$.

\section{Footprint rat behavior test}


860 The test was carried out on P20. The rats hind legs were painted with iodine and the animal was 861 allowed to move freely on a blank sheet of paper. After the rat walked along the sheet in one 862 direction, the sheet was changed. For each rat, at least five records were made. Papers with 863 footprints were scanned.

\section{Statistical analysis}

866 The values presented as means \pm standard deviations of at least three independent experiments. 867 The unpaired Student's t-test and one-way ANOVA were utilized to calculate P-values if not 868 otherwise indicated.

\section{Acknowledgements}

871 This work was supported financially by EMBO Long-Term Fellowships (MX) and grants from 872 the Swedish Research Council (ASC: 2016-02835; SS: 2015-04335), Karolinska Institutet 873 (MX, ASC, LL, IA, including an SFO Stem/Regen junior grant to ASC), Bertil Hallsten 874 Foundation (IA), EMBO Young Investigator Program (IA), Åke Wiberg Foundation (IA), 875 Chinese Scholarship Council (LL), Stiftelsen Frimurare Barnhuset i Stockholm (PN, MX), 876 Museum of the Southern Jutland (PG), National Institutes of Health (MB: NIDDK RO1 877 DK073011). The Russian Science Foundation financially supported the experiments with 878 atomic force microscopy (grant \#18-15-00401 to PT) and mechanical loading experiments 879 (grant \#19-15-00241 to ASC).

O Lambert (IRSNB), MT Olsen (ZMUC), F Zachos (NMW), PHC Lina

\section{Author Contributions}

A.S.C. designed the study. M.X. conceived the study under the guidance of A.S.C. M.X. performed all of the experiments except those specified below. J.E., P.T. and S.S. modeled the synchrotron scan data and examined SOC evolution in early tetrapods, amphibians and stem amniotes. L.L., P.N. and M.C. assisted with the biological experiments. A.K. and An.Si. assisted with salamander experiments. A.S. and K.L.C. collected material and performed Jerboa video-recording. A.P.U. and A.D.K. performed unloading experiments. I.L.A. and C.G. helped with application of the load testing instrument. P.T., E.V.M., S.K., B.S. and A.A. performed the atomic force microscopy and nanonindentation analysis. L.T.S. and M.B. performed experiments on Prx-Cre:Gsa ${ }^{\text {R201H }}$ mice. S.N. and H.M.K. contributed to experiments with SIK3 cKO mice. A.E. developed the finite element analysis. P.G., K.G., A.N.H., B.M., K.F., An.Si., H.M.K. and K.L.C. provided intellectual input. M.X. and A.S.C. wrote the paper with the help of I.A, S.S., P.G and A.N.H. All authors critically reviewed this manuscript and approved the final draft.

\section{Competing interests}

The authors declare no conflict of interests.

\section{References}


907 1. Yang L, Tsang KY, Tang HC, Chan D, Cheah KS. Hypertrophic chondrocytes 908 can become osteoblasts and osteocytes in endochondral bone formation. Proc Natl 909 Acad Sci U S A. 2014;111(33):12097-102.

910 2. Hall BK. Bones and Cartilage Developmental and Evolutionary Skeletal 911 Biology. 2 ed. San Diego: Elsevier Academic Press; 2005 Jan 2015.

912 3. Sanchez S, Tafforeau P, Ahlberg PE. The humerus of Eusthenopteron: a 913 puzzling organization presaging the establishment of tetrapod limb bone marrow. 914 Proc Biol Sci. 2014;281(1782):20140299.

915 4. Sanchez S, Tafforeau P, Clack JA, Ahlberg PE. Life history of the stem 916 tetrapod Acanthostega revealed by synchrotron microtomography. Nature. 917 2016;537(7620):408-11.

918 5. Frobisch NB, Shubin NH. Salamander limb development: integrating genes, 919 morphology, and fossils. Dev Dyn. 2011;240(5):1087-99.

920 6. Haines RW. The Primitive Form of Epiphysis in the Long Bones of 921 Tetrapods. J Anat. 1938;72(Pt 3):323-43.

922 7. Haines J-S. The evolution of epiphyses and of endochondral bone. 923 Biological Review. 1942;174:267-92.

924 8. Carter DR, Wong M. The role of mechanical loading histories in the 925 development of diarthrodial joints. J Orthop Res. 1988;6(6):804-16.

$9269 . \quad$ Castanhinha R, Araujo R, Junior LC, Angielczyk KD, Martins GG, Martins RM, 927 et al. Bringing dicynodonts back to life: paleobiology and anatomy of a new 928 emydopoid genus from the Upper Permian of Mozambique. PLoS One. 929 2013;8(12):e80974.

930 10. Chinsamy A, Abdala F. Palaeobiological implications of the bone 931 microstructure of South American traversodontids (Therapsida : Cynodontia). 932 South African Journal of Science. 2008;104(5-6):225-30.

933 11. Ray S, Chinsamy A. Diictodon feliceps (Therapsida, Dicynodontia): Bone 934 histology, growth, and biomechanics. Journal of Vertebrate Paleontology. 935 2004;24(1):180-94.

936 12. Barreto C, Albrecht RM, Bjorling DE, Horner JR, Wilsman NJ. Evidence of 937 the growth plate and the growth of long bones in juvenile dinosaurs. Science. 938 1993;262(5142):2020-3.

939 13. Horner JR, Padian, K. \& Ricqlès, A. d. . Comparative osteohistology of some 940 embryonic and perinatal archosaurs: developmental and behavioral implications 941 for dinosaurs. Paleobiology. 2001;27:39-58.

942 14. Carter DR, MikiĆ, B., Padian, K. . Epigenetic mechanical factors in the 943 evolution of long bone epiphyses. Zoological Journal of the Linnean Society 944 1998;123:163-78.

945 15. Hughes PM, Rayner, J. M. V., Jones, G. . Ontogeny of 'true'flight and other 946 aspects of growth in the bat Pipistrellus pipistrellus. Journal of Zoology. 947 1995;236:291-318.

948 16. Powers LV, Kandarian, S. C., Kunz, T. H. . Ontogeny of flight in the little 949 brown bat, Myotis lucifugus: behavior, morphology, and muscle histochemistry. 950 Journal of Comparative Physiology A: Neuroethology, Sensory, Neural, and 951 Behavioral Physiology. 1991;168:675-85.

952 17. Elangovan V, Yuvana Satya Priya E, Raghuram H, Marimuthu G. Wing 953 morphology and flight development in the short-nosed fruit bat Cynopterus 954 sphinx. Zoology (Jena). 2007;110(3):189-96. 
955 18. Wang L, Lin, A., Xiao, Y., Jiang, T., Feng, J. Postnatal development in the big956 footed bat, Myotis macrodactylus: wing morphology, echolocation calls, and flight. 957 Acta theriologica 2014;59:435-41.

958 19. Adams RA, Thibault, K. M. . Ontogeny and evolution of the hindlimb and 959 calcar: assessing phylogenetic trends. Ontogeny, functional ecology, and evolution 960 of bats. 2000:316-32.

961 20. Hermanson JW, Wilkins KT. Growth and development of two species of bats 962 in a shared maternity roost. Cells Tissues Organs. 2008;187(1):24-34.

963 21. Koyabu D, Son NT. Patterns of postcranial ossification and sequence 964 heterochrony in bats: life histories and developmental trade-offs. J Exp Zool B Mol 965 Dev Evol. 2014;322(8):607-18.

966 22. Kunz TH, Hood, W. R. . Parental care and postnatal growth in the 967 Chiroptera. Reproductive biology of bats. 2000:415-68.

968 23. Racey PA, Entwistle, A. C. . Life-history and reproductive strategies of bats. 969 Reproductive biology of bats. 2000:363-414.

970 24. Eilam D, Shefer G. The developmental order of bipedal locomotion in the 971 jerboa (Jaculus orientalis): pivoting, creeping, quadrupedalism, and bipedalism. 972 Dev Psychobiol. 1997;31(2):137-42.

973 25. Cooper LN, Sedano N, Johansson S, May B, Brown JD, Holliday CM, et al. 974 Hydrodynamic performance of the minke whale (Balaenoptera acutorostrata) 975 flipper. J Exp Biol. 2008;211(Pt 12):1859-67.

976 26. Gingerich PD, Ul-Haq M, von Koenigswald W, Sanders WJ, Smith BH, 977 Zalmout IS. New protocetid whale from the middle eocene of pakistan: birth on 978 land, precocial development, and sexual dimorphism. PLoS One. 2009;4(2):e4366. 979 27. Uhen M. Form, function, and anatomy of Dorudon atrox (Mammalia, 980 Cetacea): an archaeocete from the middle to late Eocene of Egypt. Papers on 981 Paleontology 2004;34:1-222.

982 28. Cooper LN. Forelimb anatomy. Encyclopedia of marine mammals, 2nd 983 edition. 2009:449-52.

984 29. Mellor L, Cooper, L. N., Torre, J., Brownell, Jr. R. L. . Paedomorphic 985 ossification in porpoises with an emphasis on the vaquita (Phocoena sinus). 986 Aquatic Mammals 2009;35:193-202.

987 30. Chagin AS, Karimian E, Sundstrom K, Eriksson E, Savendahl L. Catch-up 988 growth after dexamethasone withdrawal occurs in cultured postnatal rat 989 metatarsal bones. J Endocrinol. 2010;204(1):21-9.

990 31. Dupont S, Morsut L, Aragona M, Enzo E, Giulitti S, Cordenonsi M, et al. Role 991 of YAP/TAZ in mechanotransduction. Nature. 2011;474(7350):179-83.

992 32. Strano S, Munarriz E, Rossi M, Castagnoli L, Shaul Y, Sacchi A, et al. Physical 993 interaction with Yes-associated protein enhances p73 transcriptional activity. J 994 Biol Chem. 2001;276(18):15164-73.

995 33. Gerber HP, Vu TH, Ryan AM, Kowalski J, Werb Z, Ferrara N. VEGF couples 996 hypertrophic cartilage remodeling, ossification and angiogenesis during 997 endochondral bone formation. Nat Med. 1999;5(6):623-8.

998 34. Newton PT, Li L, Zhou B, Schweingruber C, Hovorakova M, Xie M, et al. A 999 radical switch in clonality reveals a stem cell niche in the epiphyseal growth plate. 1000 Nature. 2019;567(7747):234-8.

1001 35. Karaca A, Malladi VR, Zhu Y, Tafaj O, Paltrinieri E, Wu JY, et al. Constitutive 1002 stimulatory $\mathrm{G}$ protein activity in limb mesenchyme impairs bone growth. Bone. 1003 2018;110:230-7. 
1004

1005

1006

1007

1008

1009

1010

1011

1012

1013

1014

1015

1016

1017

1018

1019

1020

1021

1022

1023

1024

1025

1026

1027

1028

1029

1030

1031

1032

1033

1034

1035

1036

1037

1038

1039

1040

1041

1042

1043

1044

1045

1046

1047

1048

1049

1050

1051

36. Sasagawa S, Takemori H, Uebi T, Ikegami D, Hiramatsu K, Ikegawa S, et al. SIK3 is essential for chondrocyte hypertrophy during skeletal development in mice. Development. 2012;139(6):1153-63.

37. Xu X, Li Z, Cai L, Calve S, Neu CP. Mapping the Nonreciprocal Micromechanics of Individual Cells and the Surrounding Matrix Within Living Tissues. Sci Rep. 2016;6:24272.

38. Kleinnulend J, Veldhuijzen JP, Burger EH. Increased Calcification of Growth Plate Cartilage as a Result of Compressive Force Invitro. Arthritis Rheum-Us. 1986;29(8):1002-9.

39. Stevens SS, Beaupre GS, Carter DR. Computer model of endochondral growth and ossification in long bones: biological and mechanobiological influences. J Orthop Res. 1999;17(5):646-53.

40. Sundaramurthy S, Mao JJ. Modulation of endochondral development of the distal femoral condyle by mechanical loading. J Orthop Res. 2006;24(2):229-41.

41. Wong M, Carter DR. A Theoretical-Model of Endochondral Ossification and Bone Architectural Construction in Long-Bone Ontogeny. Anat Embryol. 1990;181(6):523-32.

42. de Medinaceli L, Freed WJ, Wyatt RJ. An index of the functional condition of rat sciatic nerve based on measurements made from walking tracks. Exp Neurol. 1982;77(3):634-43.

43. Hurlbert SH. Breeding Migrations and Interhabitat Wandering of VermilonSpotted Newt Notophthalmus-Viridescens (Rafinesque). Ecol Monogr. 1969;39(4):465-\&.

44. Cooper KL, Oh S, Sung Y, Dasari RR, Kirschner MW, Tabin CJ. Multiple phases of chondrocyte enlargement underlie differences in skeletal proportions. Nature. 2013;495(7441):375-8.

45. Breur GJ, Turgai J, Vanenkevort BA, Farnum CE, Wilsman NJ. Stereological and serial section analysis of chondrocytic enlargement in the proximal tibial growth plate of the rat. Anat Rec. 1994;239(3):255-68.

46. Wilsman NJ, Farnum CE, Leiferman EM, Fry M, Barreto C. Differential growth by growth plates as a function of multiple parameters of chondrocytic kinetics. J Orthop Res. 1996;14(6):927-36.

47. Kozhemyakina E, Lassar AB, Zelzer E. A pathway to bone: signaling molecules and transcription factors involved in chondrocyte development and maturation. Development. 2015;142(5):817-31.

48. Mehlman CT, Araghi A, Roy DR. Hyphenated history: the Hueter-Volkmann law. Am J Orthop (Belle Mead NJ). 1997;26(11):798-800.

49. Matsuda JJ, Zernicke RF, Vailas AC, Pedrini VA, Pedrini-Mille A, Maynard JA. Structural and mechanical adaptation of immature bone to strenuous exercise. J Appl Physiol (1985). 1986;60(6):2028-34.

50. Reich A, Jaffe N, Tong A, Lavelin I, Genina O, Pines M, et al. Weight loading young chicks inhibits bone elongation and promotes growth plate ossification and vascularization. J Appl Physiol (1985). 2005;98(6):2381-9.

51. Kiiskinen A. Physical training and connective tissues in young mice-physical properties of Achilles tendons and long bones. Growth. 1977;41(2):12337.

52. Biewener AA. Biomechanics of mammalian terrestrial locomotion. Science. 1990;250(4984):1097-103. 
1052 53. Butcher MT, Espinoza NR, Cirilo SR, Blob RW. In vivo strains in the femur

1053 of river cooter turtles (Pseudemys concinna) during terrestrial locomotion: tests

1054 of force-platform models of loading mechanics. J Exp Biol. 2008;211(Pt 15):2397-

1055407.

1056 54. Burdett RG. Forces predicted at the ankle during running. Med Sci Sports 1057 Exerc. 1982;14(4):308-16.

1058 55. Yadav P, Shefelbine SJ, Gutierrez-Farewik EM. Effect of growth plate 1059 geometry and growth direction on prediction of proximal femoral morphology. J 1060 Biomech. 2016;49(9):1613-9.

1061 56. Ozguven HN, Berme N. An experimental and analytical study of impact 1062 forces during human jumping. Journal of biomechanics. 1988;21(12):1061-6.

1063

1064

1065

1066

1067

1068

1069

1070

1071

1072

1073

1074

1075

1076

1077

1078

1079

1080

57. Perttunen JO, Kyrolainen H, Komi PV, Heinonen A. Biomechanical loading in the triple jump. J Sports Sci. 2000;18(5):363-70.

58. Galile G. Two new sciences, including centers of gravity \& force of percussion Translation by Stillman Drake of Galileo's 1638 Discourses and mathematical demonstrations concerning two new sciences. University of Wisconsin Press1974.

59. Campione NE, Evans DC. A universal scaling relationship between body mass and proximal limb bone dimensions in quadrupedal terrestrial tetrapods. BMC Biol. 2012;10:60.

60. ANDERSON JF. Long-bone circumference and weight in mammals, birds and dinosaurs J Zool, Lond. 1985;207:53-61.

61. Bou J, Casinos A, Ocana J. Allometry of the limb long bones of insectivores and rodents. J Morphol. 1987;192(2):113-23.

62. Biewener AA. Scaling body support in mammals: limb posture and muscle mechanics. Science. 1989;245(4913):45-8.

63. WATANABE J. Ontogeny of macroscopic morphology of limb bones in modernaquatic birds and their implications for ontogenetic ageing: Museo Argentino de Ciencias Naturales "Bernardino Rivadavia".

1081 64. Gemmell NJ, Rutherford K, Prost S, Tollis M, Winter D, Macey JR, et al. The tuatara genome reveals ancient features of amniote evolution. Nature. 2020.

1084

1085 65. Biewener AA, Thomason J, Goodship A, Lanyon LE. Bone stress in the horse forelimb during locomotion at different gaits: a comparison of two experimental methods. J Biomech. 1983;16(8):565-76.

1086 66. Biewener AA TJ, Lanyon LE. Mechanics of locomotion and jumping in the 1087 horse (Equus): in vivo stress in the tibia and metatarsus. Journal of Zoology 1088 1988;214:547-65.

1089 67. Blob RW, Biewener AA. In vivo locomotor strain in the hindlimb bones of 1090 alligator mississippiensis and iguana iguana: implications for the evolution of limb 1091 bone safety factor and non-sprawling limb posture. J Exp Biol. 1999;202 (Pt 1092 9):1023-46.

1093 68. Carrano MT, Biewener AA. Experimental alteration of limb posture in the 1094 chicken (Gallus gallus) and its bearing on the use of birds as analogs for dinosaur 1095 locomotion. J Morphol. 1999;240(3):237-49.

1096 69. RM A. Mechanics of posture and gait of some large dinosaurs. Zoological 1097 Journal of the Linnean Society. 1985;83:1-25.

1098 70. Gans C GA, Webb PW. . Vertebrate Locomotion. Dantzler WH, ed Handbook 1099 of Physiology: Section 13: Comparative Physiology, An American Physiological 1100 Society Book. 1997:55-213. 
1101 71. Ashley-Ross M. Hindlimb Kinematics during Terrestrial Locomotion in a

1102

1103

1104

1105

1106

1107

1108

1109

1110

1111

1112

1113

1114

1115

1116

1117

1118

1119

1120

1121

1122

1123

1124

1125

1126

1127

1128

1129

1130

1131

1132

1133

1134

1135

1136

1137

1138

1139

1140

1141

1142

1143

1144

1145

1146

1147

1148

1149

Salamander (Dicamptodon Tenebrosus). J Exp Biol. 1994;193(1):255-83.

72. Sanchez S, Germain D, De Ricqles A, Abourachid A, Goussard F, Tafforeau P. Limb-bone histology of temnospondyls: implications for understanding the diversification of palaeoecologies and patterns of locomotion of Permo-Triassic tetrapods. J Evol Biol. 2010;23(10):2076-90.

73. Clemente CJ, Withers PC, Thompson G, Lloyd D. Why go bipedal? Locomotion and morphology in Australian agamid lizards. J Exp Biol. 2008;211(Pt 13):2058-65.

74. Schuett GW RR, Earley RL. . The evolution of bipedal postures in varanoid lizards. Biological Journal of the Linnean Society 2009;97:652-63.

75. Biewener AA. Biomechanical consequences of scaling. J Exp Biol. 2005;208(Pt 9):1665-76.

76. Clemente CJ, Withers PC, Thompson G, Lloyd D. Evolution of limb bone loading and body size in varanid lizards. J Exp Biol. 2011;214(Pt 18):3013-20.

77. JD C. Bones: structure and mechanics. Princeton University Press. 2002.

78. JC H. Body size, spatial distribution, and microhabitat use in the caimans, Melanosuchus niger and Caiman crocodilus, in a Peruvian lake. Journal of Herpetology 1994;28:508-13.

79. McNease L JT. A study of immature alligators on Rockefeller Refuge, Louisiana, Division of Administration, Administrative Services. 1989.

80. Tucker AD MH, Limpus CJ. . Habitat use by Crocodylus johnstoni in the Lynd River, Queensland. Journal of Herpetology 1997;31:114-21.

81. ML. D. Sur les épiphyses des lacertiliens. Zoologischer Anzeiger 1884;7:65-

70.

82. Sanchez S, Klembara J, Castanet J, Steyer JS. Salamander-like development in a seymouriamorph revealed by palaeohistology. Biol Lett. 2008;4(4):411-4.

83. Haines RW. The structure of the epiphyses in Sphenodon and the primitive form of secondary centre. J Anat. 1939;74(Pt 1):80-90.

84. DR C. Ontogenetic limits on locomotor performance. Physiological zoology 1996;69:467-88.

85. Marsh RL. Ontogenesis of contractile properties of skeletal muscle and sprint performance in the lizard Dipsosaurus dorsalis. J Exp Biol. 1988;137:11939.

86. RB H. Phylogenetic and ontogenetic determinants of sprint performance in some diurnal Kalahari lizards. Koedoe. 1982;25:43-8.

87. T GJ. Ontogenetic and individual variation in size, shape and speed in the Australian agamid lizard Amphibolurus nuchalis. Journal of Zoology. 1985;207:425-39.

88. Butcher MT, Blob RW. Mechanics of limb bone loading during terrestrial locomotion in river cooter turtles (Pseudemys concinna). J Exp Biol. 2008;211(Pt 8):1187-202.

89. Walker WF, Jr. A structural and functional analysis of walking in the turtle, Chrysemys picta marginata. J Morphol. 1971;134(2):195-214.

90. Zani PA GJ, Kram R. Giant Galápagos tortoises walk without inverted pendulum mechanical-energy exchange. Journal of Experimental biology. 2005;208:1489-94.

91. Nakajima Y HR, Endo H. . Turtle humeral microanatomy and its relationship to lifestyle. Biological Journal of the Linnean Society 2014;112(719-734). 
1150 92. de Oliveira CA, Nogueira JC, Mahecha GA. Sequential order of appearance

1151 of ossification centers in the opossum Didelphis albiventris (Didelphidae) 1152 skeleton during development in the marsupium. Ann Anat. 1998;180(2):113-21.

1153 93. Geiger M, Forasiepi AM, Koyabu D, Sanchez-Villagra MR. Heterochrony and 1154 post-natal growth in mammals--an examination of growth plates in limbs. J Evol 1155 Biol. 2014;27(1):98-115.

1156 94. Winters LM, Green, W. W., Comstock, R. E. Prenatal Development of the 1157 Bovine. University of Minnesota Agricultural Experiment Station. 1942.

1158 95. Libbin RM, Mitchell OG, Guerra L, Person P. Delayed carpal ossification in 1159 Notophthalmus viridescens Efts: Relation to the progress of mesopodial 1160 completion in newt forelimb regenerates. J Exp Zool. 1989;252(2):207-11.

1161 96. Grigelioniene G, Suzuki HI, Taylan F, Mirzamohammadi F, Borochowitz ZU, 1162 Ayturk UM, et al. Gain-of-function mutation of microRNA-140 in human skeletal 1163 dysplasia. Nat Med. 2019;25(4):583-+.

1164 97. Bassett JH, Williams GR. Role of Thyroid Hormones in Skeletal 1165 Development and Bone Maintenance. Endocr Rev. 2016;37(2):135-87.

1166 98. Xing W, Cheng S, Wergedal J, Mohan S. Epiphyseal chondrocyte secondary 1167 ossification centers require thyroid hormone activation of Indian hedgehog and 1168 osterix signaling. J Bone Miner Res. 2014;29(10):2262-75.

1169 99. Desjardin C, Charles C, Benoist-Lasselin C, Riviere J, Gilles M, Chassande O, 1170 et al. Chondrocytes play a major role in the stimulation of bone growth by thyroid 1171 hormone. Endocrinology. 2014;155(8):3123-35.

1172 100. Williams GR. Thyroid hormone actions in cartilage and bone. Eur Thyroid 1173 J. 2013;2(1):3-13.

1174 101. Das B, Heimeier RA, Buchholz DR, Shi YB. Identification of direct thyroid 1175 hormone response genes reveals the earliest gene regulation programs during 1176 frog metamorphosis. J Biol Chem. 2009;284(49):34167-78.

1177 102. Sanchez S, Ahlberg PE, Trinajstic KM, Mirone A, Tafforeau P. Three1178 dimensional synchrotron virtual paleohistology: a new insight into the world of 1179 fossil bone microstructures. Microsc Microanal. 2012;18(5):1095-105.

1180 103. Paganin D, Mayo SC, Gureyev TE, Miller PR, Wilkins SW. Simultaneous 1181 phase and amplitude extraction from a single defocused image of a homogeneous 1182 object. J Microsc. 2002;206(Pt 1):33-40.

1183 104. Labiche JC, Mathon O, Pascarelli S, Newton MA, Ferre GG, Curfs C, et al. 1184 Invited article: the fast readout low noise camera as a versatile x-ray detector for 1185 time resolved dispersive extended x-ray absorption fine structure and diffraction 1186 studies of dynamic problems in materials science, chemistry, and catalysis. Rev Sci 1187 Instrum. 2007;78(9):091301.

1188 105. Metscher BD. MicroCT for comparative morphology: simple staining 1189 methods allow high-contrast 3D imaging of diverse non-mineralized animal 1190 tissues. BMC Physiol. 2009;9:11.

1191 106. Ovchinnikov DA, Deng JM, Ogunrinu G, Behringer RR. Col2a1-directed 1192 expression of Cre recombinase in differentiating chondrocytes in transgenic mice. 1193 Genesis. 2000;26(2):145-6.

1194 107. Chagin AS, Vuppalapati KK, Kobayashi T, Guo J, Hirai T, Chen M, et al. G1195 protein stimulatory subunit alpha and Gq/11alpha G-proteins are both required 1196 to maintain quiescent stem-like chondrocytes. Nat Commun. 2014;5:3673. 


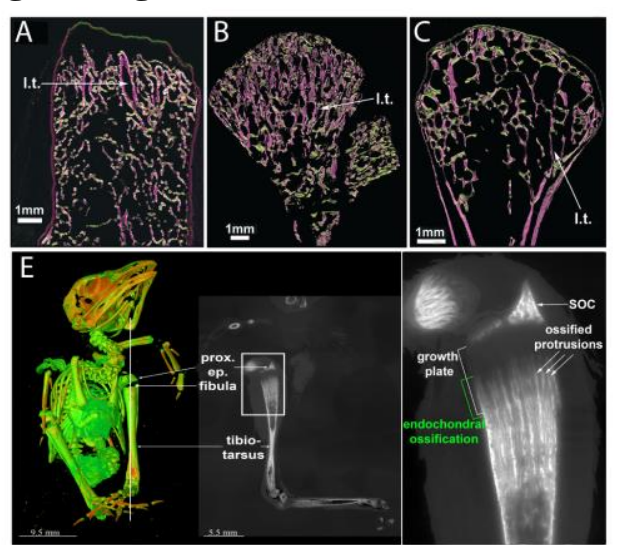

F Without SOC and without protrusions in hypertrophic zone (i.e. fetal tetrapods and urodeles)
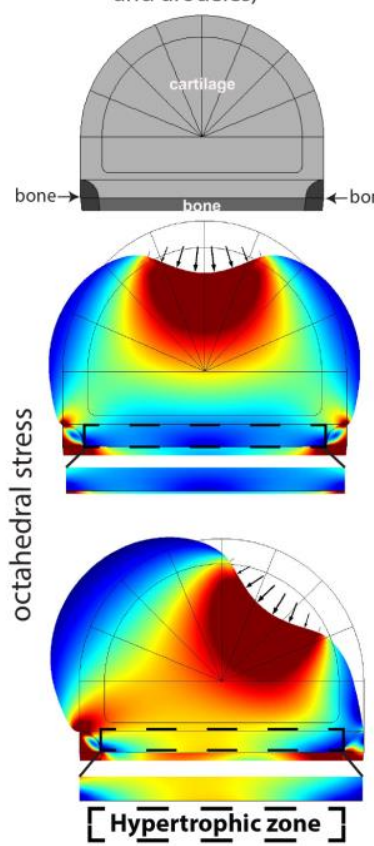
trophic zone
(i.e. synapsids, including mammal)

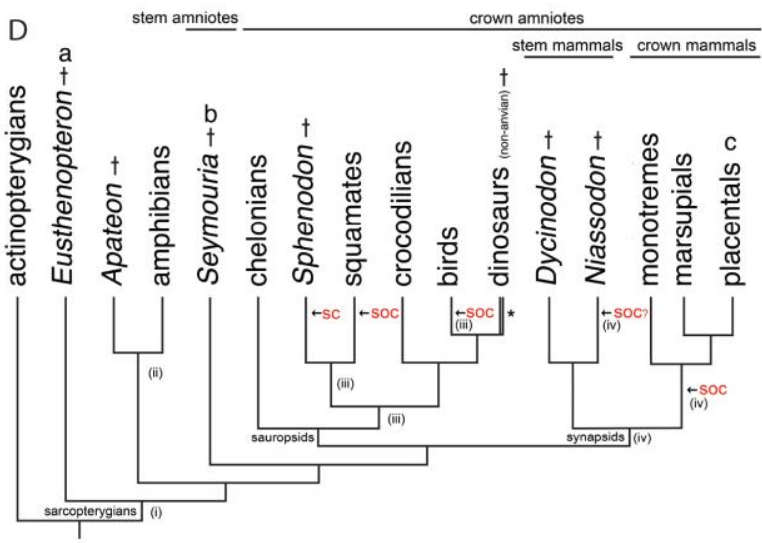

Without SOC and with protrusions

(i.e. stem tetrapods, chelonians, crocodilians)

Without $\mathrm{SOC}$ and with extended protrusions (i.e. dinosaurs, including
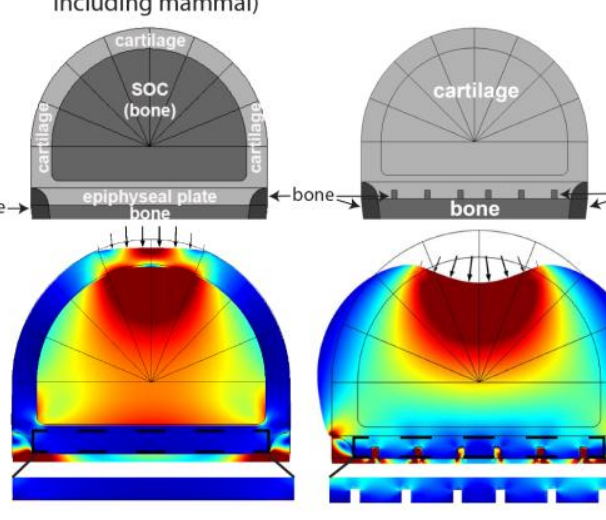
birds)
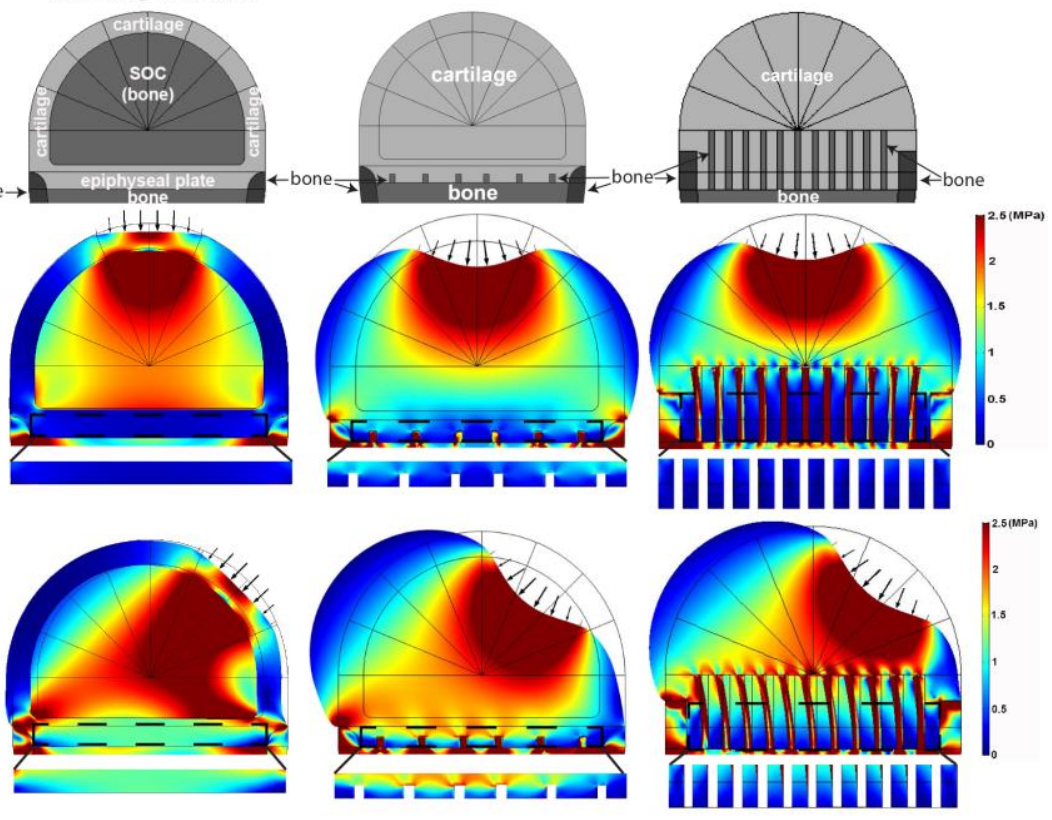

1200

Figure 1. Evolutionary appearance of the SOC and its role in distributing octahedral stress.

(A-C) The proximal humeral metaphyses of Eusthenopteron (A), Seymouria (B) and Sciurus vulgaris (red squirrel) (C). The longitudinal trabeculae resulting from endochondral ossification at the base of the growth plate in adults are shown in pink, while the transverse trabeculae appear green. The longitudinal trabeculae (1.t.) are designated marrow processes invading the growth plate. (D) Phylogeny tree, illustrating the major changes in the evolution of long-bone epiphyses. The black arrows indicate the presence of a secondary calcified center (SC) or a secondary ossification center (SOC). (i) Estimated appearance of endochondral formation of long bones, based on the fossil record to date; (ii) Cartilaginous epiphyses in long-bones; (iii) Hypothetical presence of an ancestral SCs in sauropsids; (iv) Hypothetical presence of an ancestral SOCs in synapsids. * indicates paraphyly within the group. (E) A synchrotron scan of a juvenile sparrow demonstrates the presence of SOC. 1, a virtual thin section of the proximal epiphysis. prox. ep., proximal epiphysis. (F) Deformation and distribution of octahedral stress from FEA simulation in different evolutionary taxa. Columns refer to (from left to right) "without SOC" (representing i.e., fetal tetrapods and urodeles), "with SOC" (representing i.e., 
1217 synapsids including mammal), "stem tetrapods" (representing i.e., juvenile stem tetrapods, chelonians, crocodilians) and archosaurs (representing i.e., birds and non-avian dinosaurs). For comparison of structural functionality, all situations used the same basic geometry and materials. The small arrows indicate the direction of loading. Supra-physiological loading level (3 MPa) was modeled. Hypertrophic zone free of bony elements is presented under each model 1222 for direct comparison.
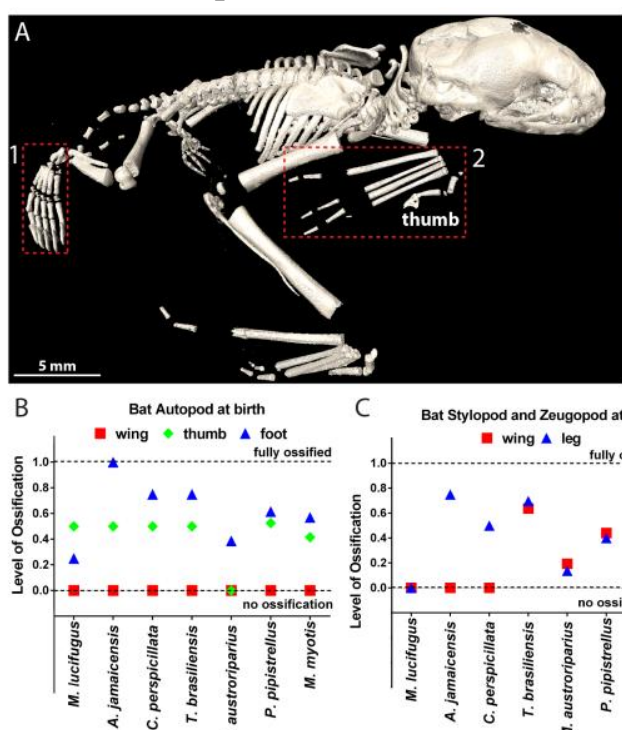

$<$ i
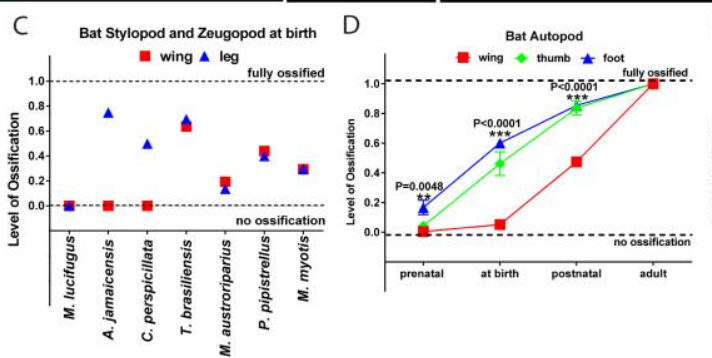
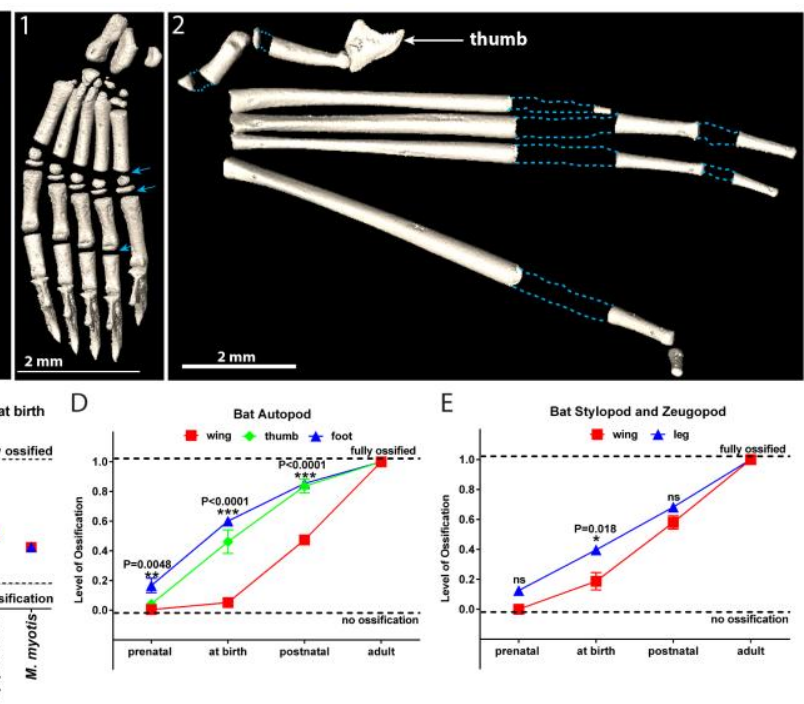

Figure 2. Comparative analysis in bats.

1225

(A) microCT image of a 1-3-days-old bat Pipistrellus pipistrellus. (1) foot, with arrows pointing to growth plates, (2) wing, with dashed blue lines outlining the areas of non-ossified cartilage. (B-C) Ossification of the autopod (B) and of the stylopod and zeugopod (C) in 7 bat species at birth. (D-E) Generalized pattern of ossification of the autopod (D) and stylopod and zeugopod (E) of 6 bat species combined (Myotis lucifugus, Artibeus jamaicensis, Carollia perspicillata, Tadarida brasiliensis, Myotis austroriparius and Myotis myotis). 


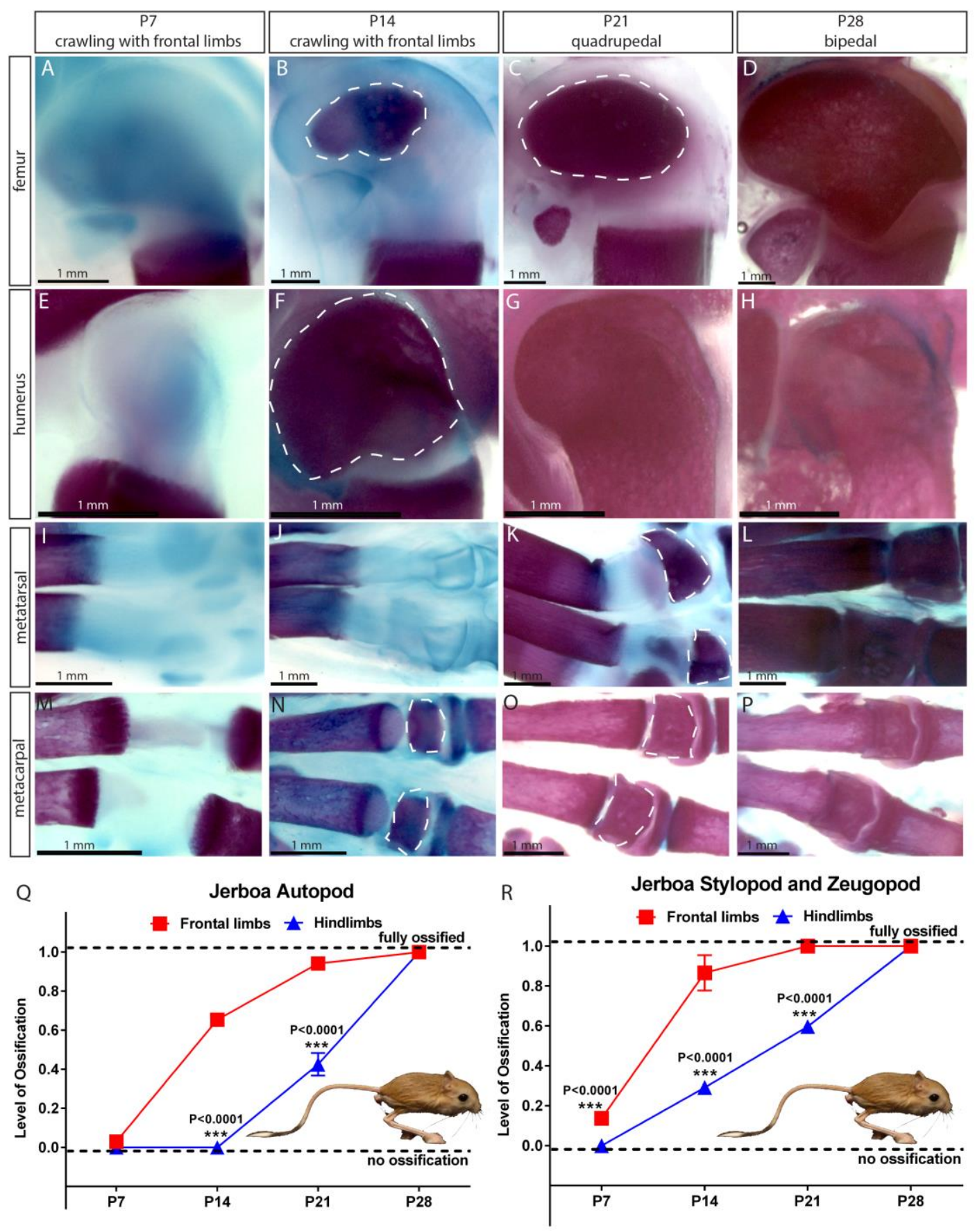

(A-P) Alcian blue and alizarin red staining of femur (A-D), humerus (E-H), metatarsal (I-L) and metacarpal (M-P) of Jaculus jaculus (three-toed jerboa) at various postnatal (P) time points. The dashed white lines outline the SOC. The gait pattern was derived from our weekly observations (see Video S3). (Q-R) Ossification of the autopod (Q) and stylopod and zeugopod (R) of Jaculus jaculus (three-toed jerboa) at various postnatal stages. Data are means $\pm \mathrm{SD}$, representing inter-individual variation, One-way ANOVA. In (Q-R), $n=2$ jerboa analyzed. $n s$, not significant. 
A

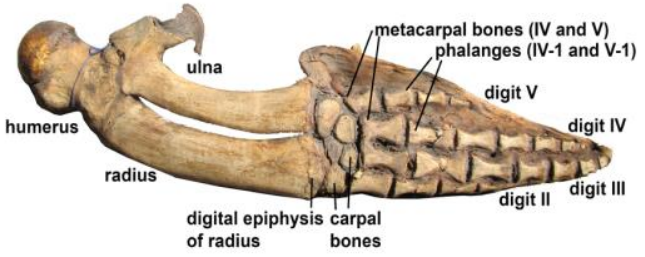

B
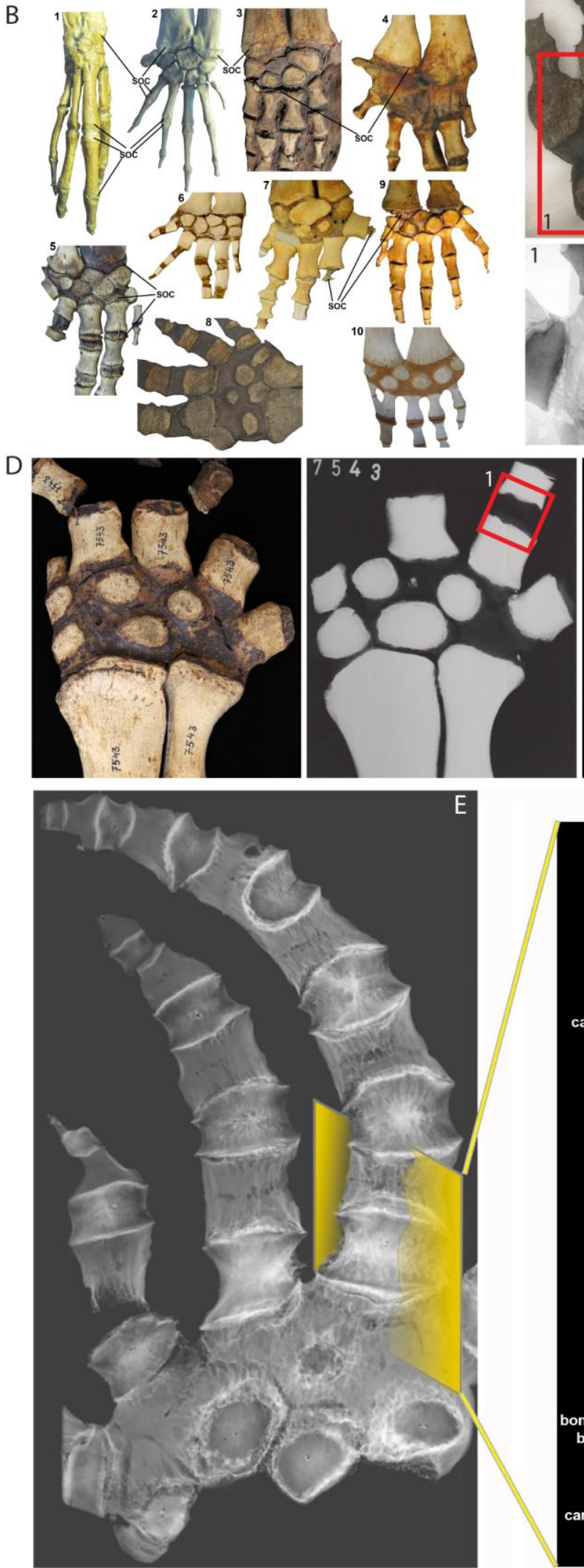
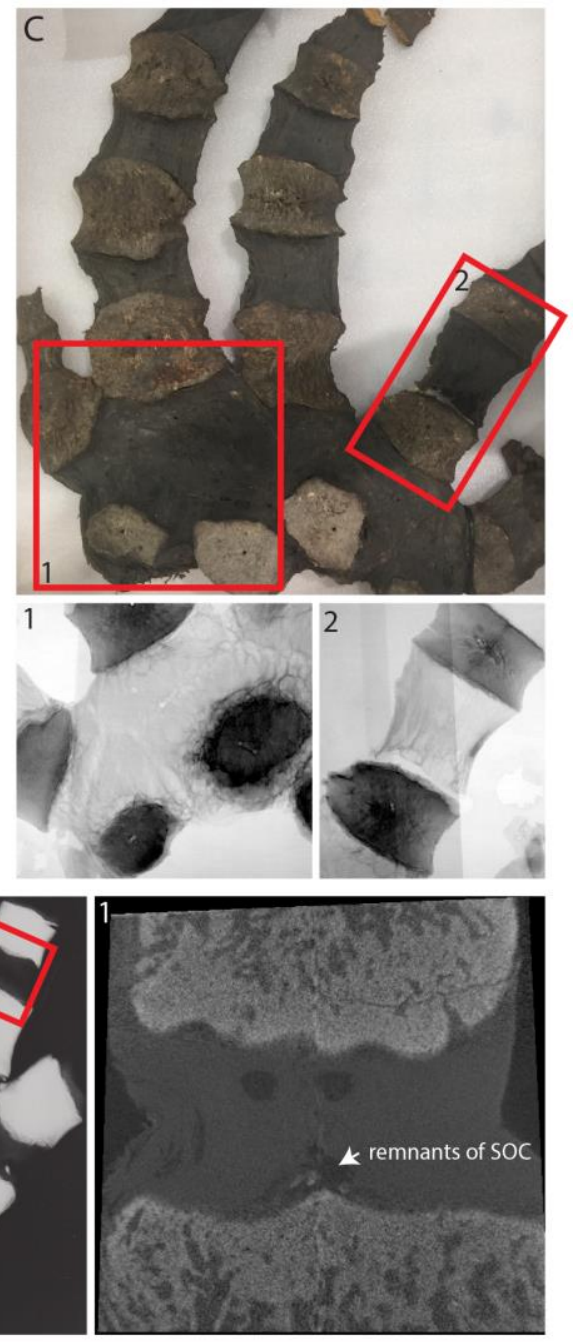

Sagittal section

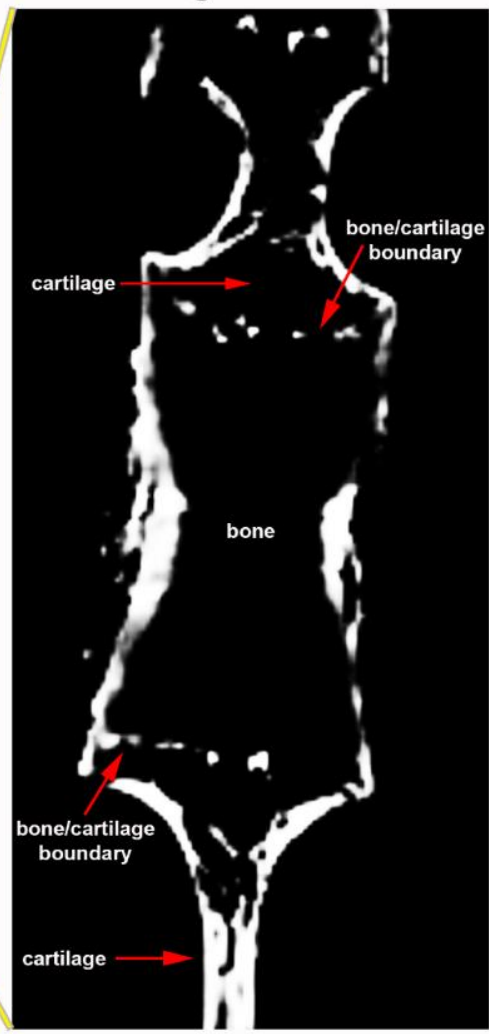




\section{Figure 4. Comparative analysis in whales.}

(A) Forelimb bones of the minke whale Balaenoptera acutorostrata. (B) Comparative anatomy of bony and cartilaginous epiphyses in the distal portion of the forelimbs of various cetacean whales: B1-B2 - early cetaceans; B3-B4 - baleen whales; B5-B10 - toothed whales. (B1) Maiacetus inuus, semi-aquatic from Eocene era (56-33.9 Mya), (B2) Dorudon atrox, fullyaquatic from Eocene era (B3) minke whale Balaenoptera acutorostrata from Miocene era (23-5.3 Mya) (B4) North Atlantic right whale Eubalaena glacialis, (B5) pilot whale Globicephala melas (B6) boto river dolphin Inia geoffrensis, (B7) narwhal Monodon monoceros, (B8) killer whale Orcinus orca, (B9) juvenile and (B10) subadult sperm whale Physeter catodon. (C) Sample image of an adult killer whale Orcinus orca (same species as B8) with X-ray images of the highlighted areas $(1,2)$ from the corresponding image on their left. (D) Sample (left) and X-ray (right) images of an adult beluga whale Delphinapterus leucas. (D1) Micro-CT reconstruction of the highlighted phalange joint. (E) CT image of an adult killer whale Orcinus orca and a virtual sagittal section of the metacarpal II demonstrating the absence of the SOC.
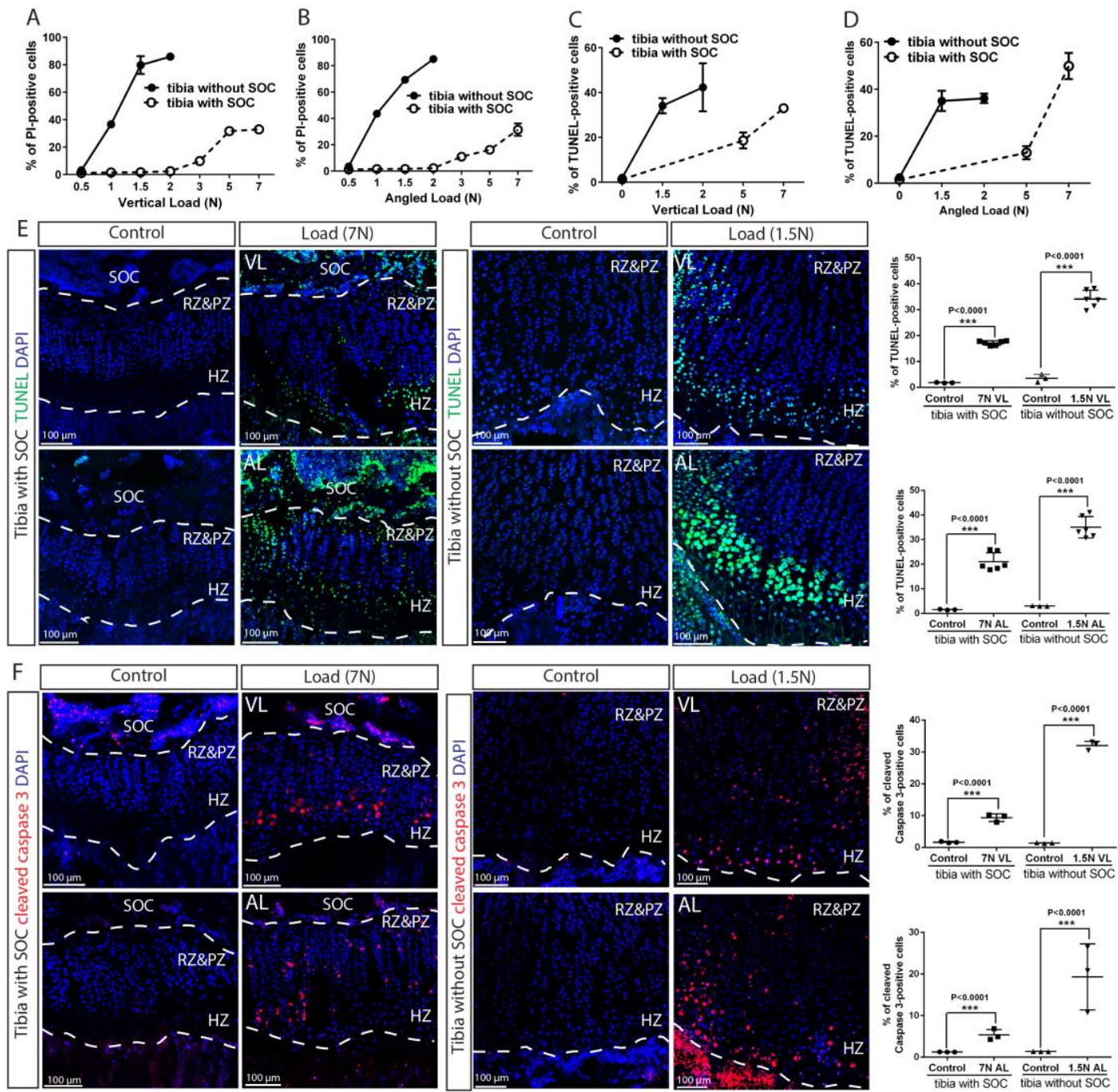

Figure 5. The SOC protects epiphyseal chondrocytes from apoptosis induced by mechanical stress.

(A-D) Quantification of propidium iodide- (PI) (A-B) and TUNEL- (C-D) positive cells in the growth plates of tibia with and without SOCs loaded vertically $(\mathrm{A}, \mathrm{C})$ or at an angle (B, D). (E- 
1262 Caspase 3 staining (red) (F) in the growth plates of loaded tibias with and without SOCs. DAPI was used for counterstaining (blue). Data are means \pm SD, two-tailed Student's $t$ test. In (A-F), $\mathrm{n}=3$. The control and loaded tibia were from the same animal. VL, vertical load, AL, angled load. RZ\&PZ, resting zone \& proliferative zone combined, HZ, hypertrophic zone. "tibia with SOC" and "tibia without SOC" in the figure refer to tibias from 30-day-old mice and 10-day-

1267 old rats respectively (see Figure S3A-B).

A

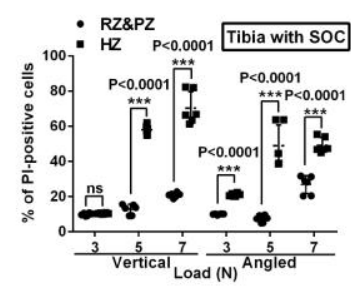

E
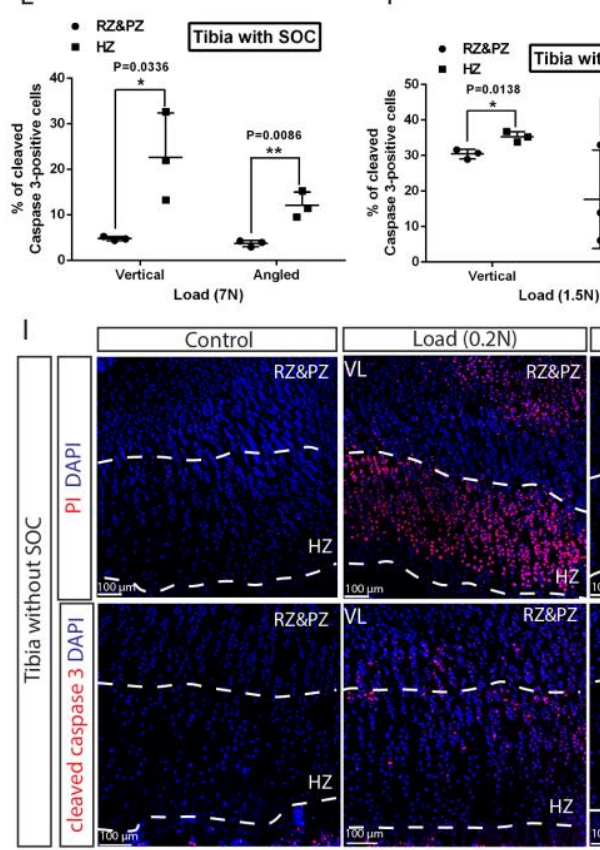

B

$\mathrm{F}$
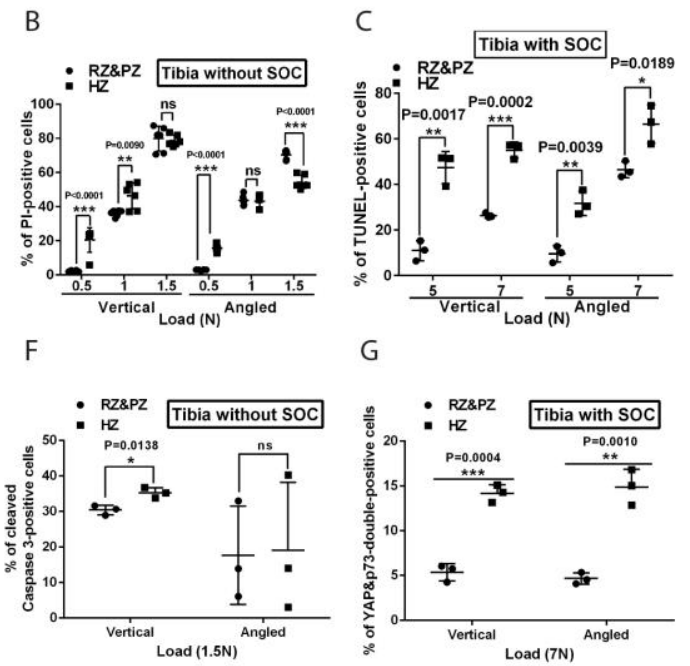

G

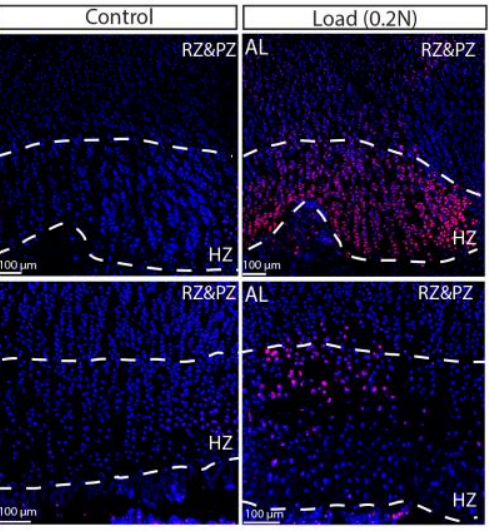

D

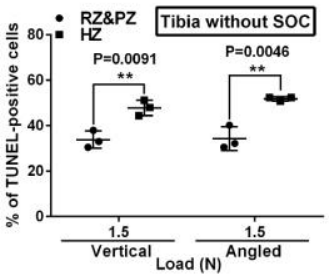

$\mathrm{H}$
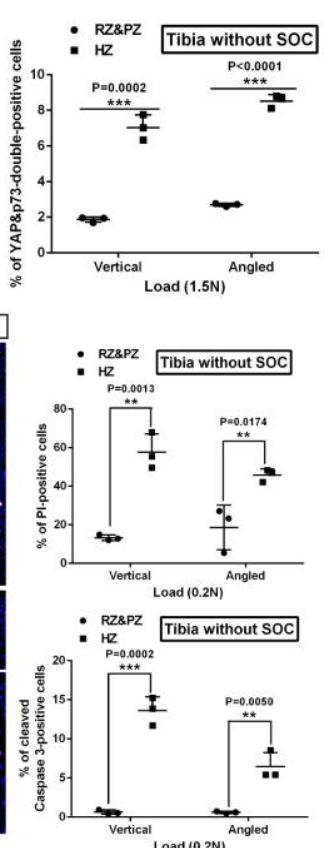

1268

1269

1270

1271

1272

1273

1274

1275

1276

1277

1278

1279

1280

1281

1282

Figure 6. Hypertrophic cells are the most sensitive cell type in response to mechanical stress.

(A-D) Comparison of the extent of cell death between resting+proliferative zones (RZ\&PZ) and hypertrophic zone $(\mathrm{HZ})$ of the vertically and angularly loaded growth plates with $(\mathrm{A}, \mathrm{C})$ and without (B, D) SOCs as assessed by PI staining (A-B) and TUNEL (C-D). (E to H) Distribution of immunohistological staining along longitudinal axis for cleaved Caspase-3 staining (E-F) and YAP\&p73 double staining (G-H) of the vertically and angularly loaded tibia with $(\mathrm{E}, \mathrm{G})$ and without $(\mathrm{F}, \mathrm{H})$ SOCs. (I) Representative images and quantification of propidium iodide (PI) staining (upper panel) and cleaved Caspase 3 staining (lower panel) in the growth plates of vertically or angularly $0.2 \mathrm{~N}$ loaded tibias without SOCs. The control and loaded tibia were from the same animal. DAPI was used for counterstaining (blue) in (I). Data are means $\pm S D$, two-tailed Student's t test. In (A-I), n=3. ns, not significant. "tibia with SOC" and "tibia without SOC" refer to tibias from 30-day-old mice and 10-day-old rats respectively (see Figure S3A-B). 
1283

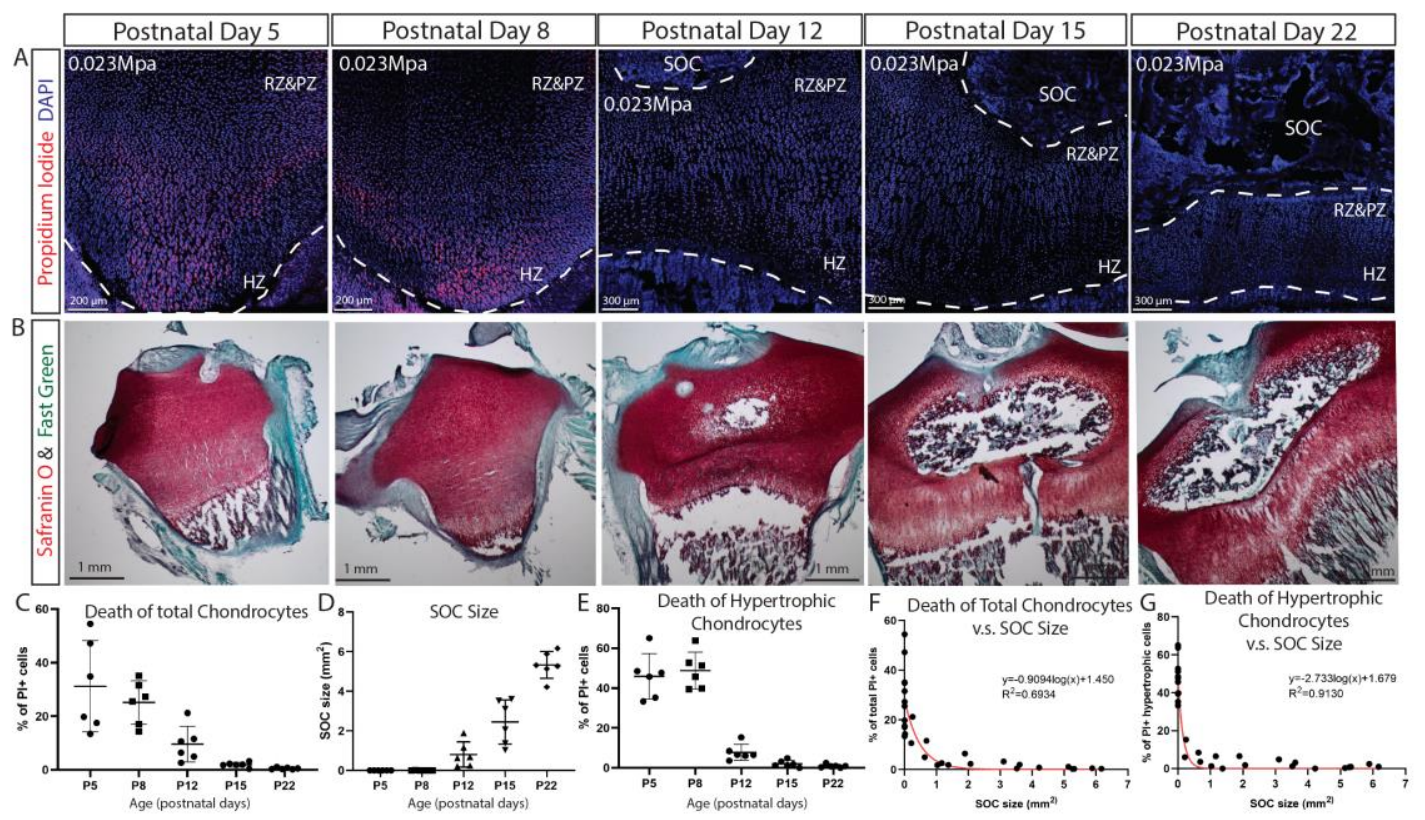

Figure 7. SOC size is negatively correlated with load-induced chondrocyte death in rat tibia growth plate.

(A) Representative images of the distribution of propidium iodide (PI)-positive cells in the growth plates of rat tibia with the same vertical pressure of $0.023 \mathrm{Mpa}$ (equivalent to the load of $0.2 \mathrm{~N}$ for postnatal day 5 rat tibia). (B) Representative images of safranin $\mathrm{O}$ and fast green staining of the corresponding rat tibia to illustrate the development of SOC. (C-E) Quantification of PI-positive total growth plate chondrocytes (C), SOC size (D) and PI-positive hypertrophic chondrocytes (E) in the loaded rat tibia at different ages. (F-G) Correlation analysis of PI-positive total growth plate chondrocytes (F) or PI-positive hypertrophic chondrocytes $(\mathrm{G})$ with SOC size. Semilog line of nonlinear regression curve fit was used (red line). During the end of culture period bones were incubated with PI, which penetrates only into dead cells (red). DAPI (blue) counterstaining of all nucleus was done on the fixed tissue sections. RZ\&PZ, resting zone \& proliferative zones, HZ, hypertrophic zone. Data are means $\pm \mathrm{SD}, \mathrm{n}=6$. Two independent experiments were performed with 3 animals per time point per experiment. Each data point in (F-G) represents one animal. 

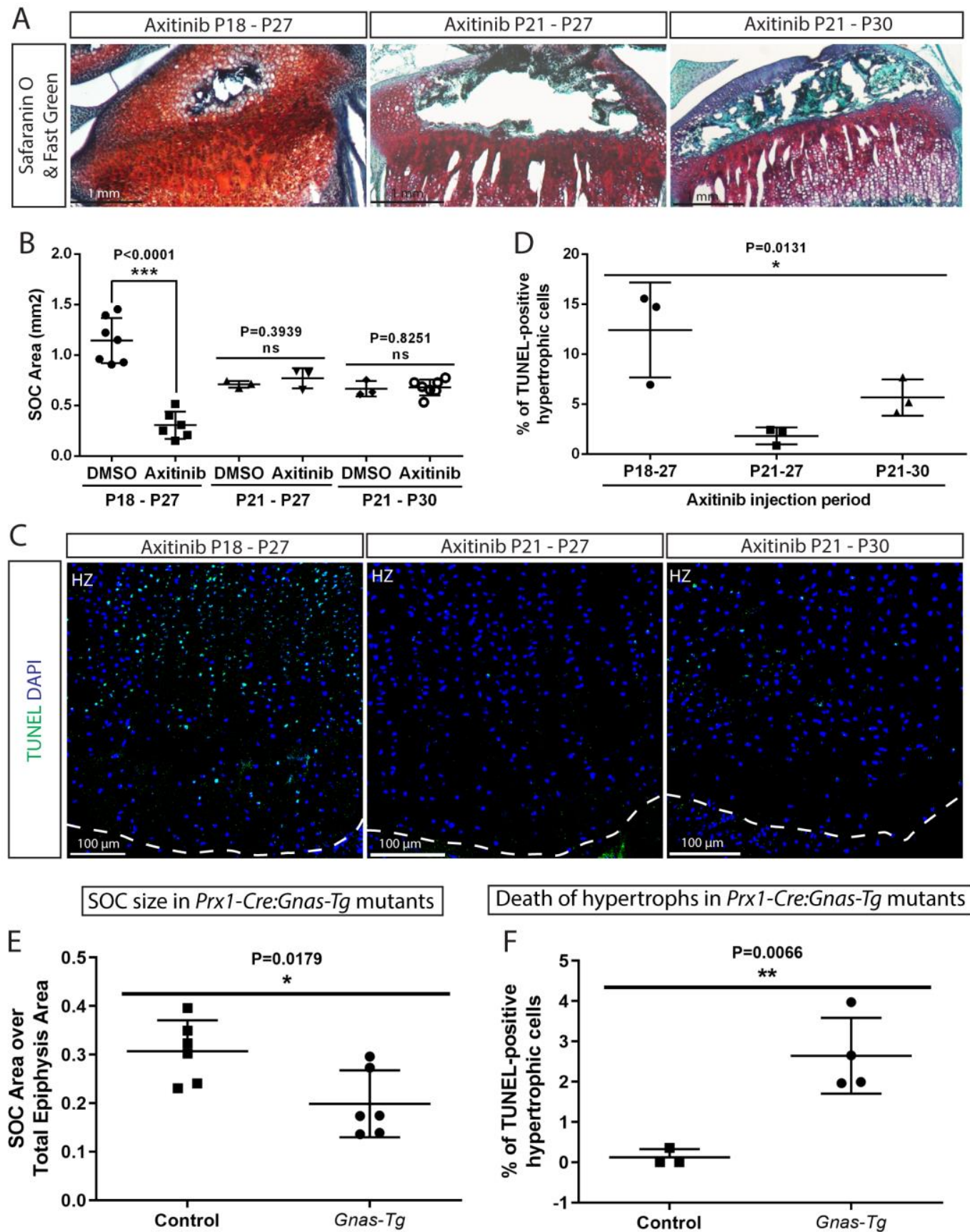

Death of hypertrophs in Prx1-Cre:Gnas-Tg mutants

Figure 8. Inhibition of the SOC size in vivo is associated with increased epiphyseal chondrocyte apoptosis.

Size of the SOC was inhibited either pharmacologically employing axitinib (A-D) or genetically employing activation of Gnas (E, F). (A-B) Representative histology images of the tibia epiphysis (A) and quantification of the SOC size (B) of wild type mice injected with Axitinib at various time periods. P refers to postnatal day. (C-D) Representative images (C) and quantification of TUNEL staining (green) (D) in the hypertrophic cells of the tibia growth plate of the Axitinib injected mice. (E-F) Quantification of SOC size (E) and TUNEL staining (F) of the hypertrophic cells in the growth plates of stop Gnas ${ }^{R 201 H}$ (Control) and PrxCre:Gnas ${ }^{R 201 H}$ (Gnas-Tg) mice. DAPI was used for counterstaining (blue) in (C). Data are means $\pm \mathrm{SD}$, two-tailed Student's $t$ test. In $(\mathrm{A}-\mathrm{F}), \mathrm{n}=3$. ns, not significant. 
A

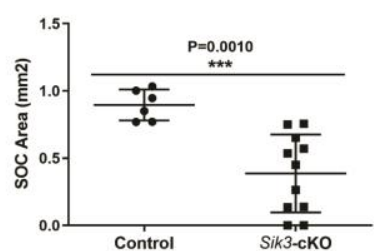

C

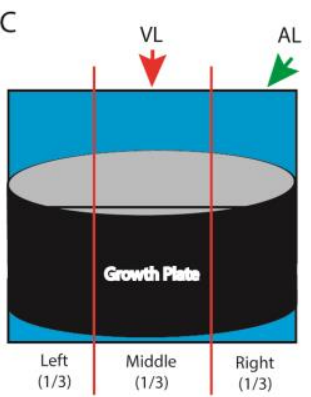

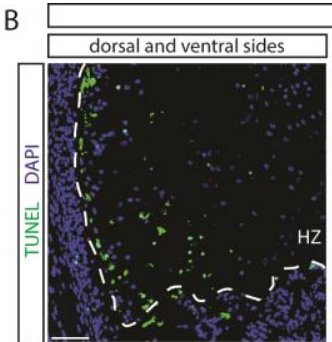

D
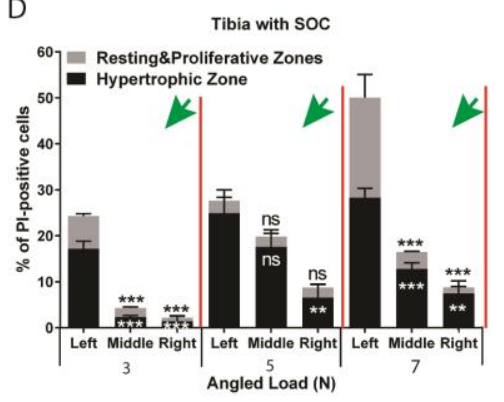

Angled Load (N)

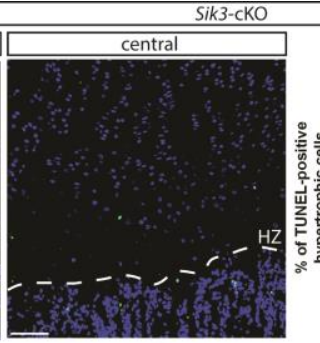

E

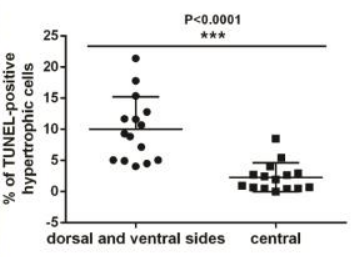

Tibia without SOC

$\left.{ }^{60}\right]=$ Resting\&Proliferative Zones

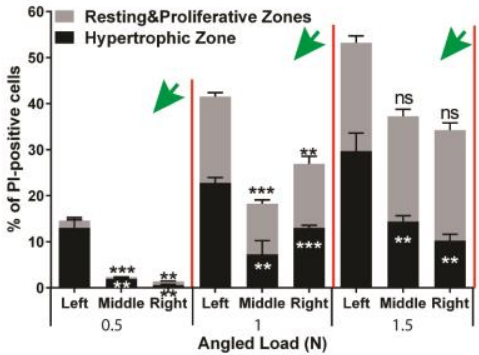

Angled Load (N)
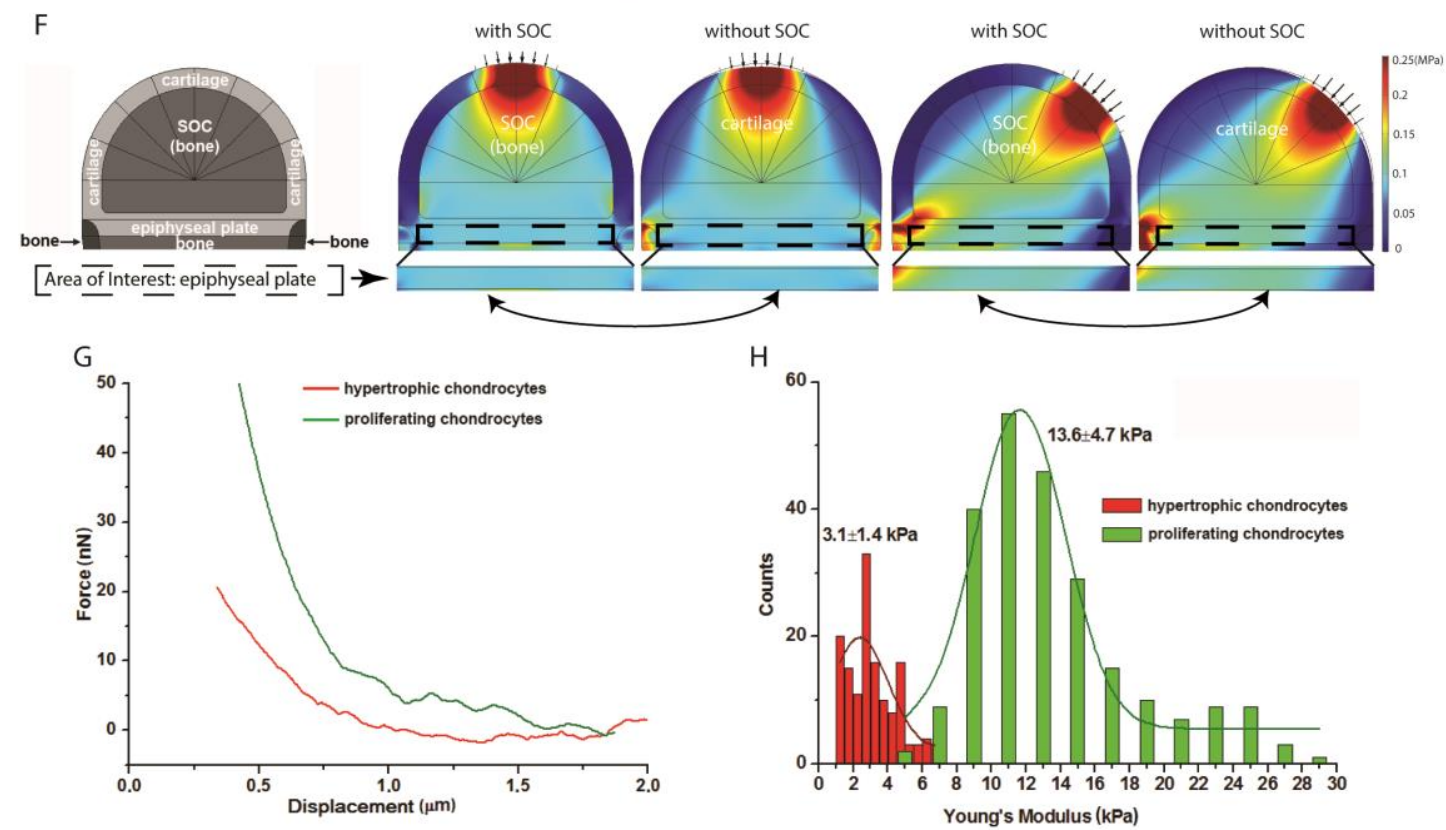

Figure 9. Low stiffness of hypertrophic chondrocytes renders them sensitive to mechanical stress.

(A) Quantification of the SOC size of postnatal day $28 \mathrm{Sik}^{\mathrm{F} / F}$ (Control) and Col2al-Cre:Sik $3^{\text {F/F }}$ (Sik3-cKO) mice. (B) Representative images and quantification of TUNEL staining (green) between the lateral and middle parts of Sik3-cKO mice. DAPI was used for counterstaining (blue). HZ, hypertrophic zone. In (A-B), data are means \pm SD, two-tailed Student's $t$ test, $n=3$ for "Control" and $\mathrm{n}=5$ for "Sik3-cKO". (C) Schematic illustration of a section plan and loading directions in relation to the quantitative analysis of cell death distribution in the lateral-medial direction presented in (D-E). The growth plate is divided into three equal parts, (left, middle and right) in relationship to the angle at which the load is applied (green or red arrows). (D-E) Quantification of propidium iodide (PI)-positive chondrocytes in the resting\&proliferative zones and hypertrophic zone of tibias with (D) and without (E) SOCs subjected to an angled load (AL, green arrow, always applied from the right). Data are means $\pm \mathrm{SD}$, One-way ANOVA. **, $\mathrm{P}<0.001$ and $* * *, \mathrm{P}<0.0001$ in comparison with the left portion. ns, not significant. The black and white asterisks indicate the significance for the resting\&proliferative and hypertrophic zones, respectively. (F) Principal compressive stress from FEA modeling with 
physiological loading level. The small arrows indicate the direction of loading. The two-headed curved arrows indicate areas for comparison. (G) Typical force curves for hypertrophic and proliferating chondrocytes obtained by atomic force microscopy. (H) Comparison of the elastic moduli of hypertrophic and proliferating chondrocytes.
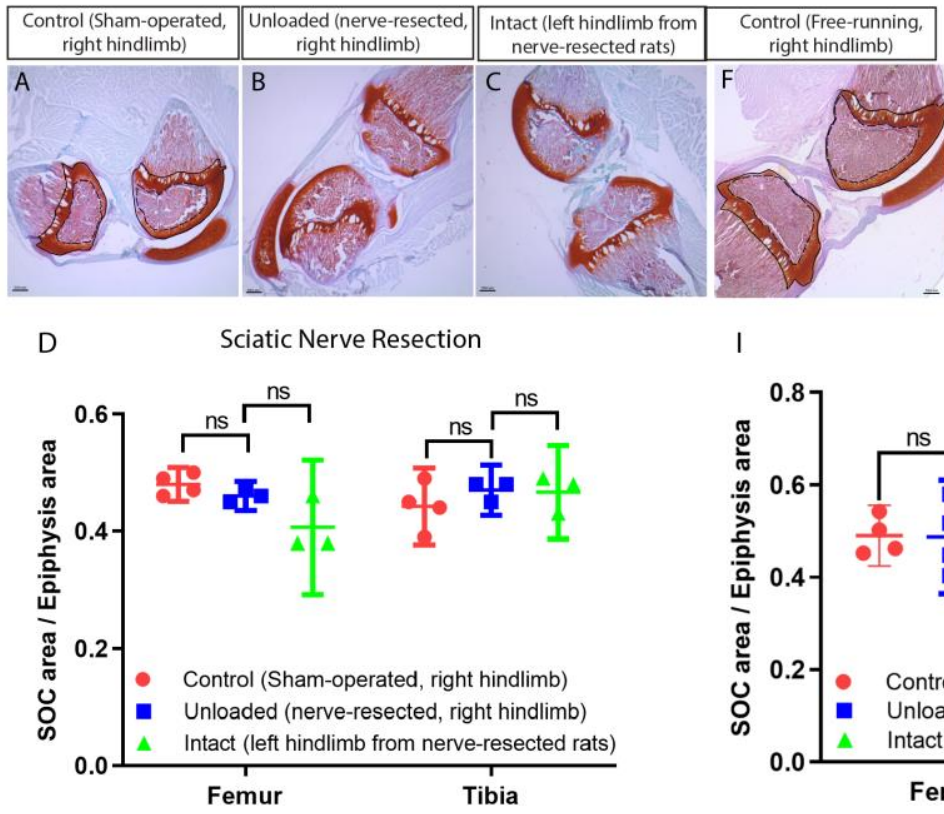

E

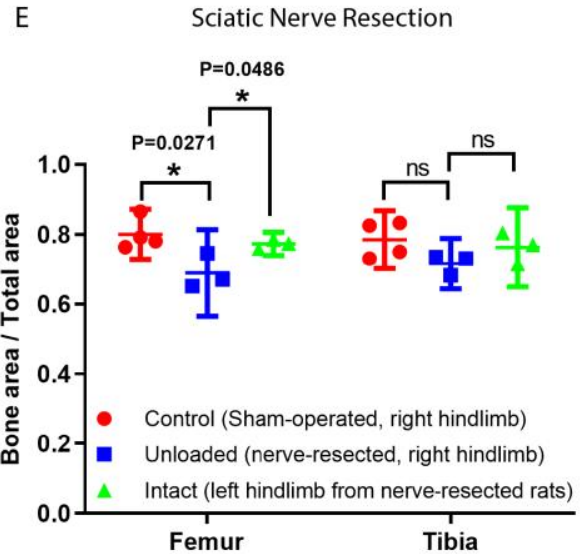

I

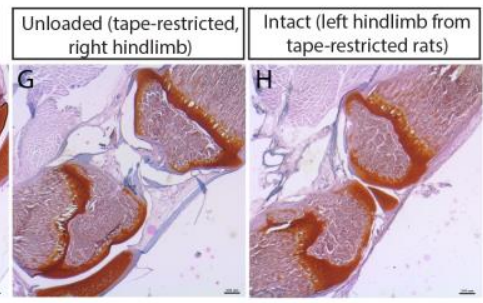

Tape Restriction
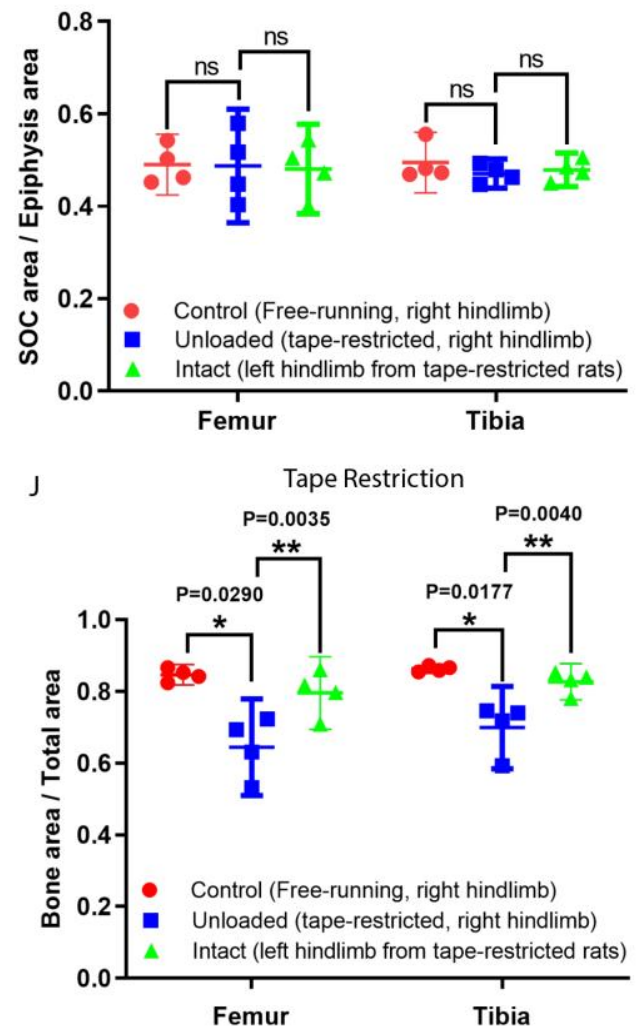

1332

Figure 10. Unloading of the hindlimbs in growing rats by either sciatic nerve resection or by tape restriction.

Sciatic nerve resection (A-E) or leg restriction with a kinesiology tape (F-J) was done at 10 days of age and both hindlimbs (manipulated and intact) were analyzed at day 23. For (A-E) sham-operated littermates were used as a control (only right hindlimb was utilized), whereas for (F-J) unmanipulated littermates were used as a control (only right hindlimb was utilized). (A-C, F-H) Representative Safranin O and Fast Green stained images of the knee joint. Black dash lines outline the SOC and solid lines outline the epiphysis area in A and F. Quantification of SOC size (normalized to total epiphysis size) (D, I) and bone mass (within the primary spongiosa region) (E, J). Bars are means $\pm 95 \%$ confidential interval and every dot represent individual animal, two-tailed Student's t test. ns, not significant. 

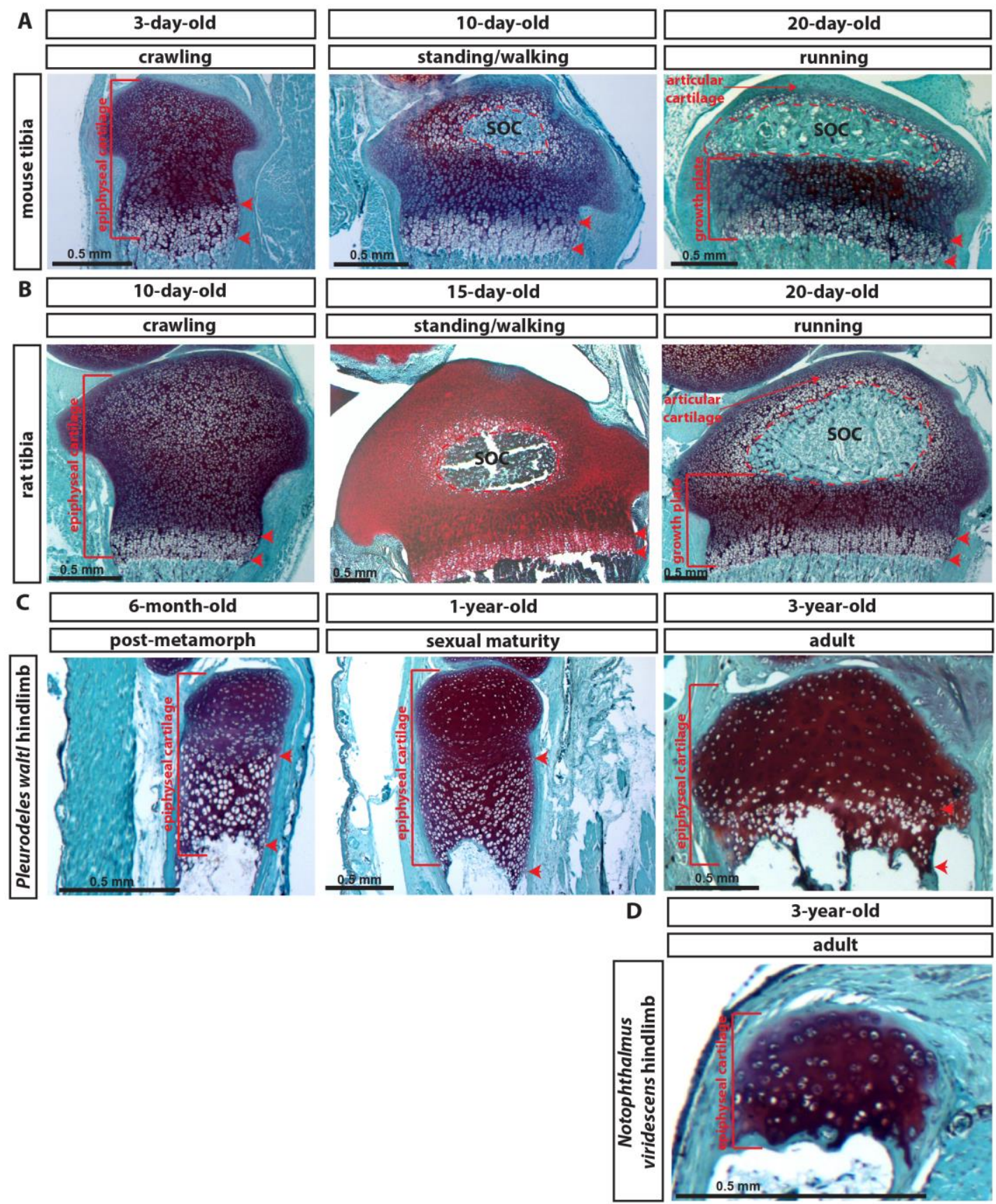

Figure 1-figure supplement 1. Development of articular and epiphyseal cartilage in rodents and salamanders.

1347 (A-D) Representative safranin O and fast green staining of sections of the epiphyseal end of the tibia of mouse (A), rat (B), and the hind limb of salamander species Pleurodeles waltl (C) and Notophthalmus viridescens (D). The dashed red lines outline the SOC. Red arrow heads mark the zone of hypertrophic cells. Walking behavior is indicated according to our daily observations. Pleurodeles waltl is a Iberian newt that spends most their life in water. Notophthalmus viridescens is a north American newt that has an aquatic larval, a terrestrial eft and a semiaquatic adult stage. 


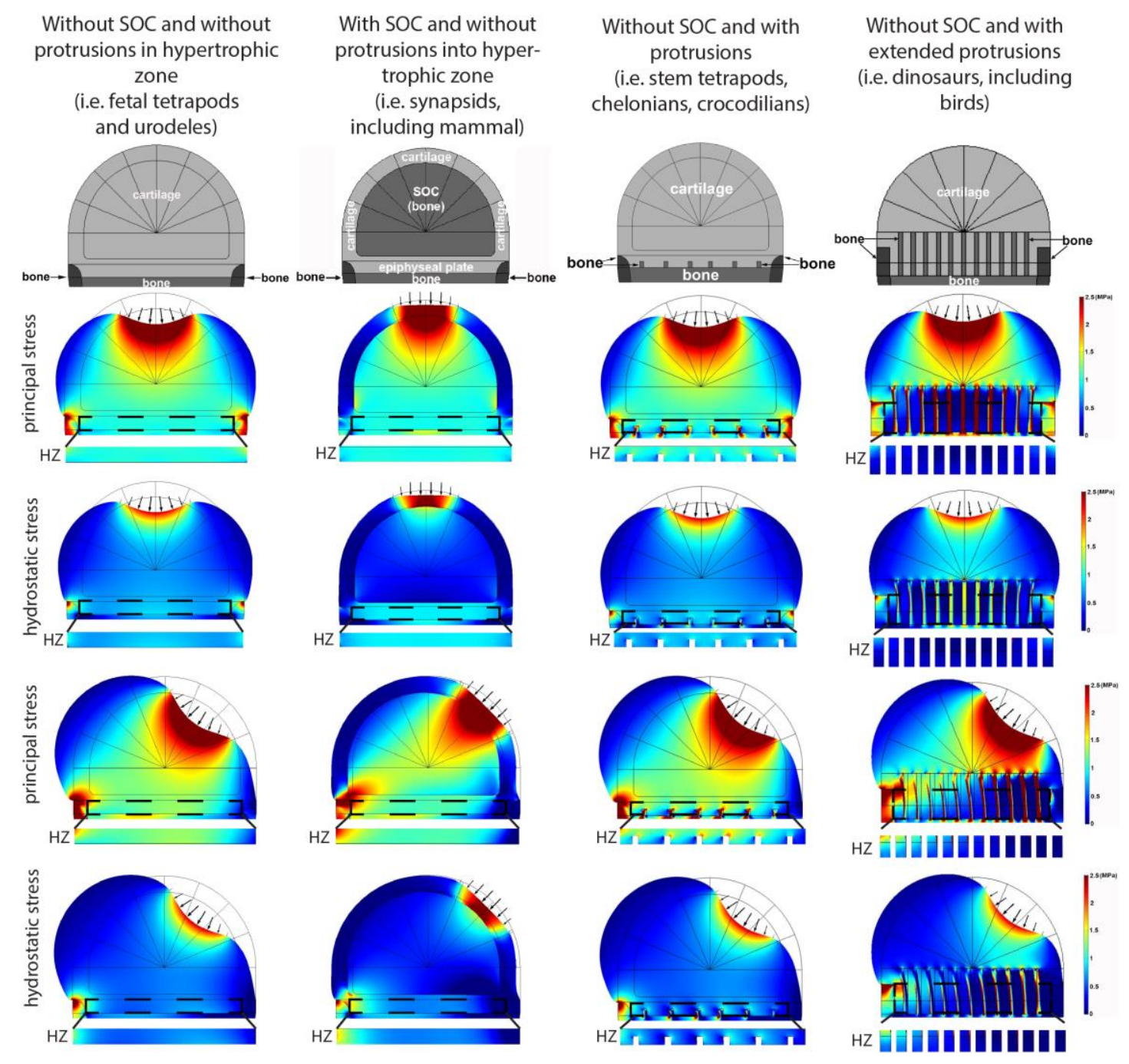

Figure 1-figure supplement 2. Deformation and distribution of comparison stresses from FEA simulation in different evolutionary taxa. Columns refer to (from left to right) "without SOC" (representing i.e., fetal tetrapods and urodeles), "with SOC" (representing i.e., synapsids including mammal), "stem tetrapods" (representing i.e., juvenile stem tetrapods, chelonians, crocodilians) and archosaurs (representing i.e., birds and non-avian dinosaurs). For comparison of structural functionality, all situations used the same basic geometry and materials. The small arrows indicate the direction of loading. Supra-physiological loading level (3 MPa) was modeled. Hypertrophic zone free of bony elements is presented under each model for direct comparison. Hypertrophic zone, HZ. 

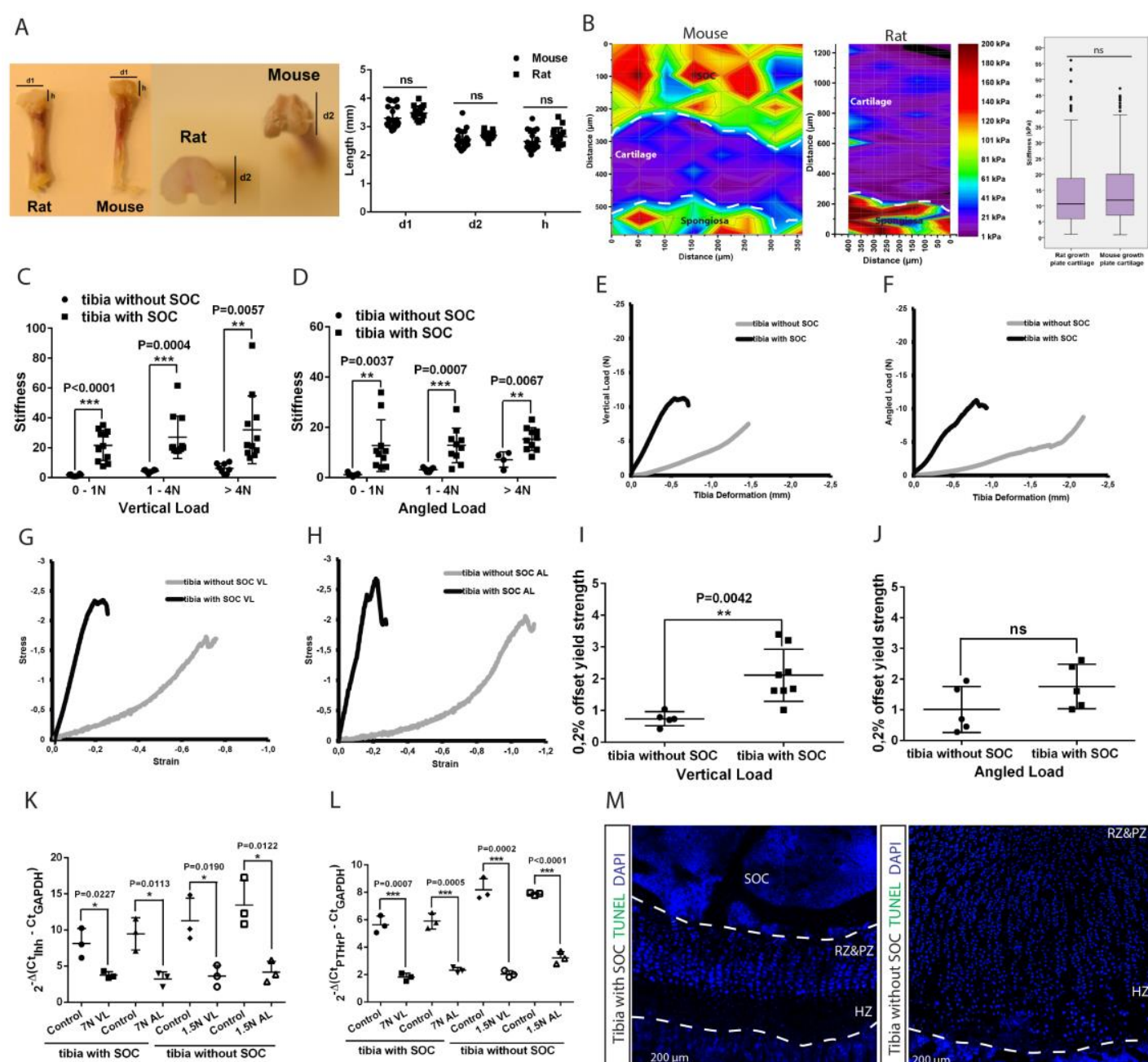

$\mathrm{L}$ M
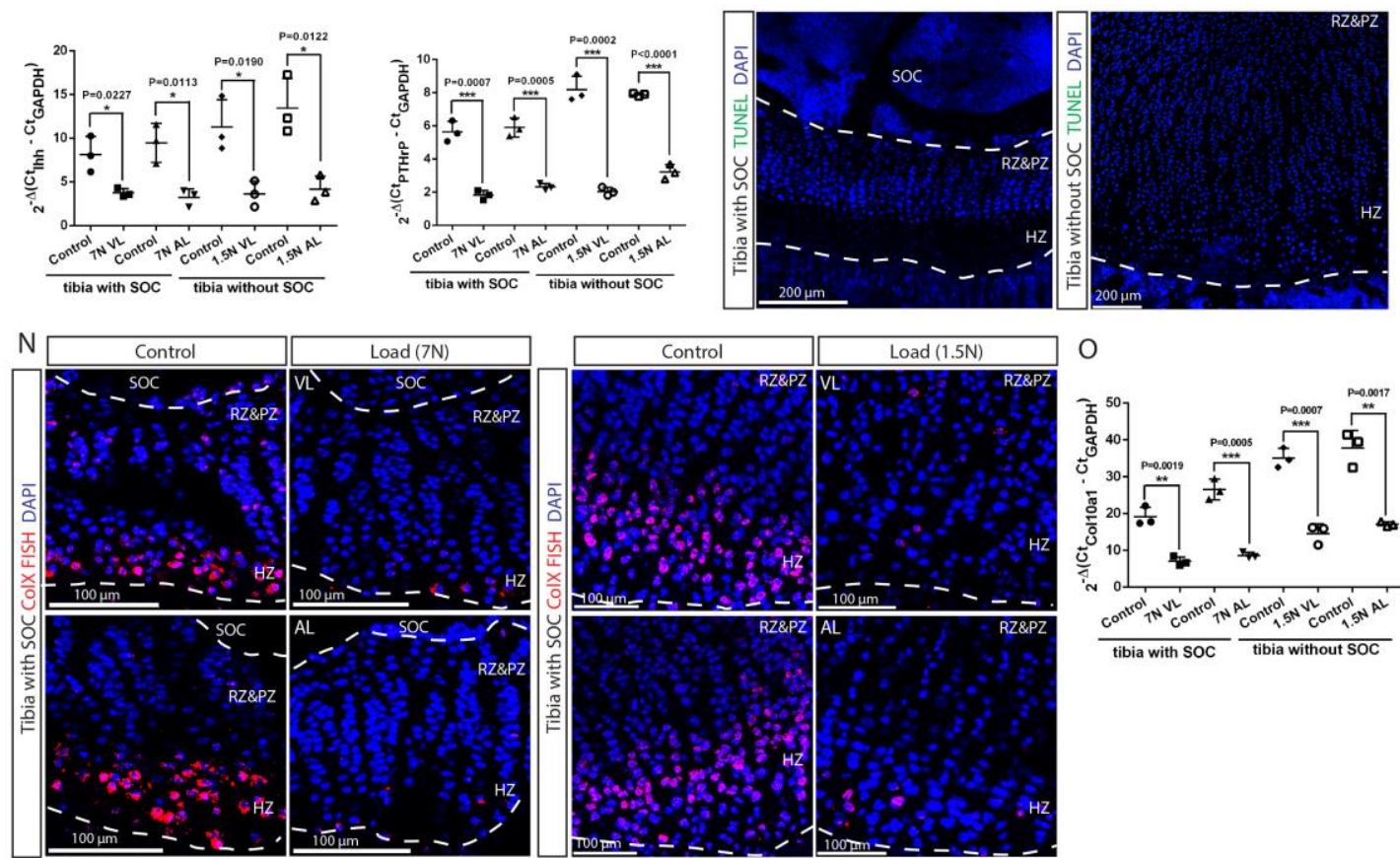

Figure 5-figure supplement 3. (A) Mouse (30-day-old) and rat (10-day-old) tibia with and without an SOC, respectively, have similar physical dimensions of the epiphyses (d1, d2, h). (B) Representative images and quantification of the nanoindentation test of 30-day old mice and 10-day-old rats. (C-D) The stiffness of bones with fully developed SOCs (tibia with SOC) and with immature SOCs (tibia without SOC) was measured at different loads and in both the vertical (C) and angled (D) direction. (E-J) Representative load/deformation (E-F) and stress/strain (G-H) curves of vertically (VL) (E, G) and angularly (AL) (F, H) loaded tibias with and without SOCs. (I-J) Elasticity of tibias with and without SOCs under vertical (I) and angled load (J). (K-L) QPCR analysis of Indian hedgehog (Ihh) (K) and parathyroid hormone related 
1375 Representative images of growth plates of tibias with and without SOCs fixed after dissection and prior loading. TUNEL staining (green) and nucleus counterstaining with DAPI (blue). (N) Fluorescent in situ hybridization of Collagen type X (ColX) in the loaded growth plates of tibias with and without SOCs. VL, vertical load, AL, angled load. RZ\&PZ, resting zone \& proliferative zones, HZ, hypertrophic zone. (O) QPCR analysis of ColX level in the epiphyseal end of tibia with and without SOCs exposed to different loading. Data are means $\pm S D$, twotailed Student's t test, except in (B) where Mann-Whitney U test was used. In (A), n=23 for "Mouse" and $\mathrm{n}=15$ for "Rat". In (B), $n=5$ for "Mouse" and $n=4$ for "Rat". In (C), n=10 for "tibia with SOC" at all loads; $n=9$ for "tibia without SOC" at $0-1 \mathrm{~N}$ and $1-4 \mathrm{~N}, \mathrm{n}=4$ for $>4 \mathrm{~N}$. In (D), $n=11$ for "tibia with SOC" at all loads; $n=8$ for "tibia without SOC" at all loads. In (I-L, O), $n=3$. ns, not significant. 

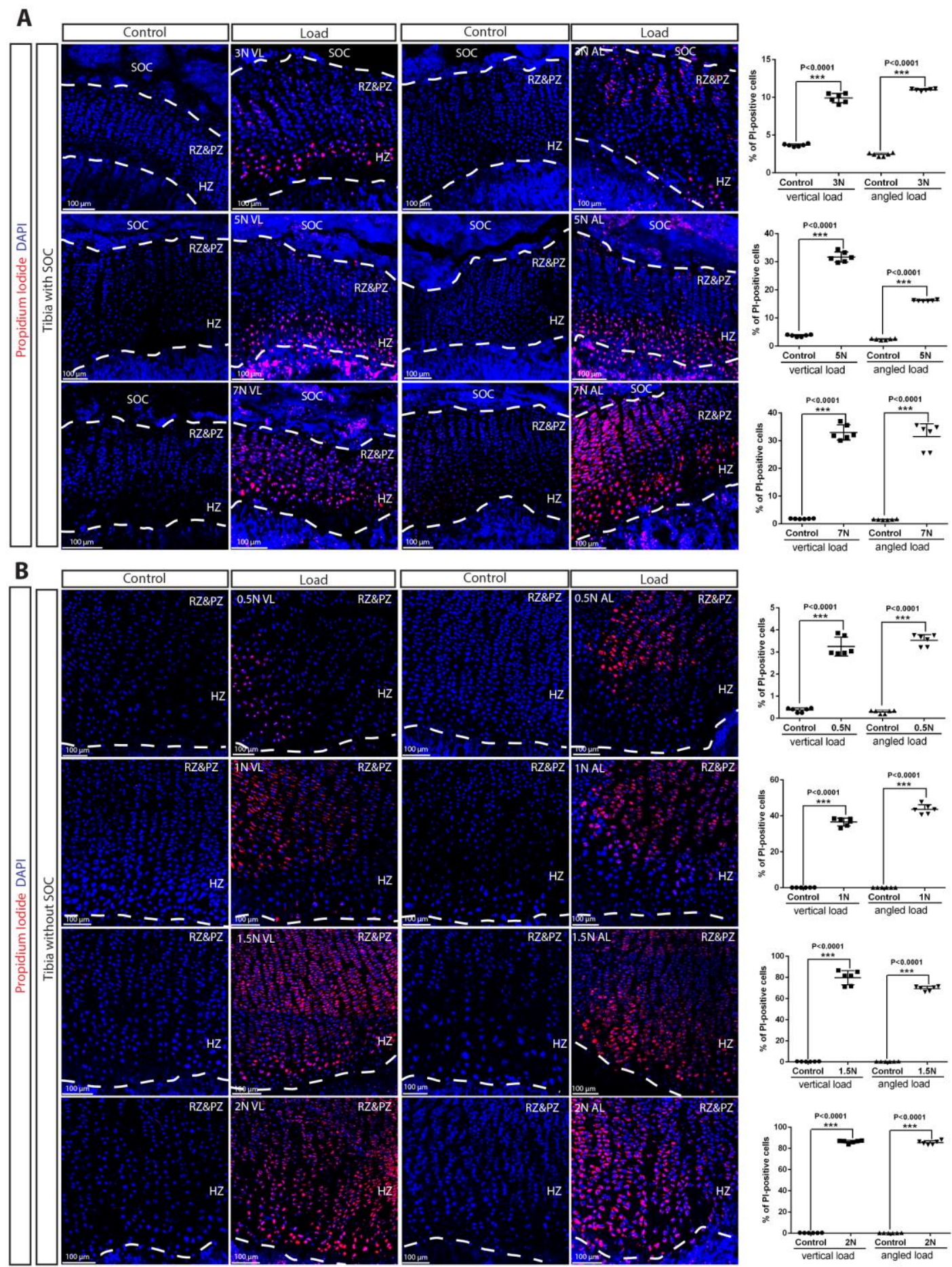

Figure 5-figure supplement 4. Death of the growth plate chondrocytes upon various loading in the presence and absence of the SOC. (A-B) Representative images and quantification of the distribution of propidium iodide (PI)-positive cells in the growth plates of tibia with (A) and without a SOC (B) loaded vertically or angularly. During the end of culture period bones were incubated with PI, which penetrates only into dead cells (red). Representative pictures (images) and quantifications (graphs) are shown. DAPI (blue) counterstaining of all nucleus was done on the fixed tissue sections. RZ\&PZ, resting zone \& proliferative zones, HZ, hypertrophic zone. "tibia with SOC" and "tibia without SOC" refer to tibias from 30-day-old mice and 10-day-old rats respectively (see Figure S3A-B). Data are means \pm SD, two-tailed Student's t test. In (AB), $n=3$. 

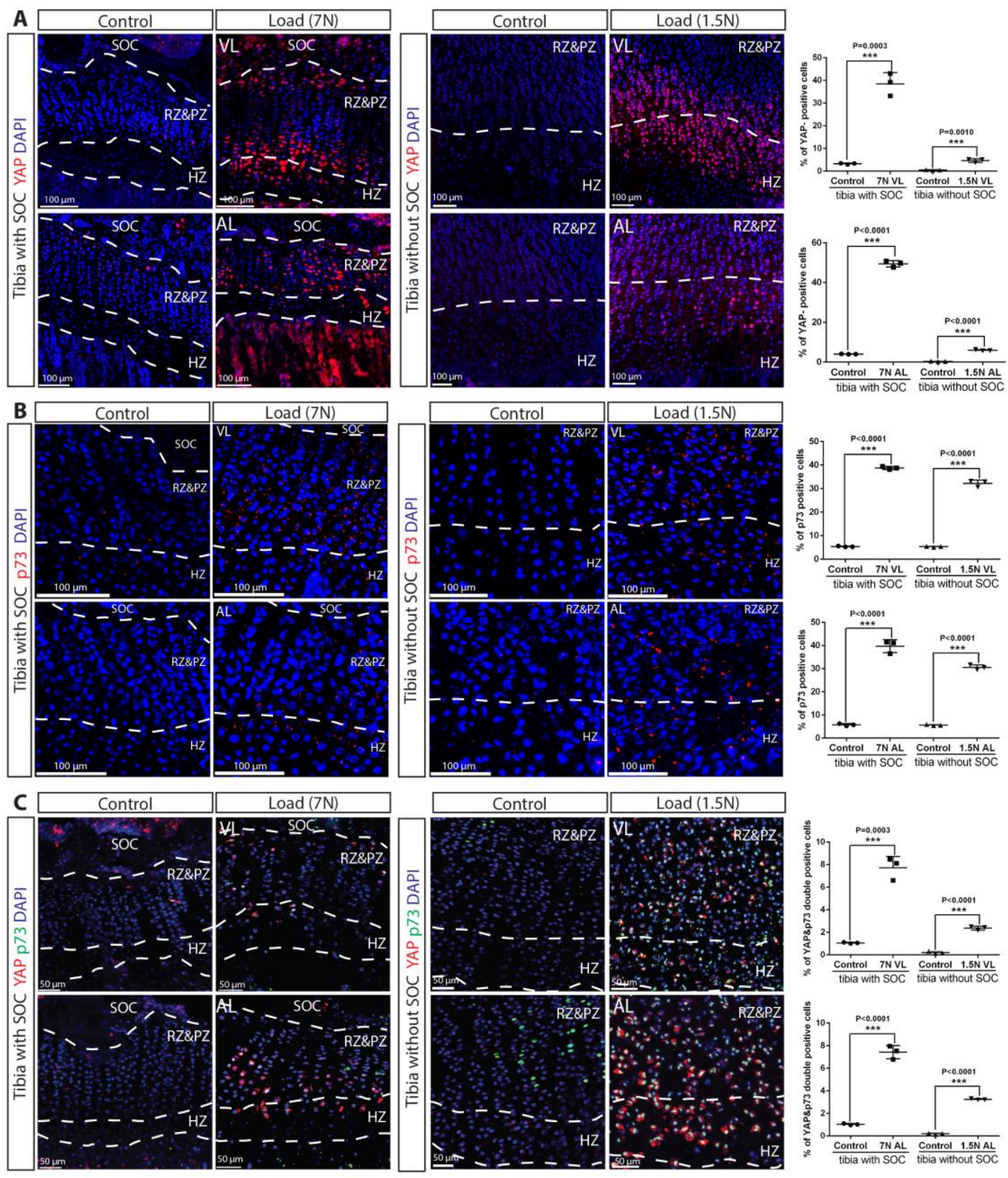

Figure 5-figure supplement 5. (A-C) Immunostaining and quantification of YAP (A), p73 (B) and $\mathrm{YAP}+\mathrm{p} 73$ double positive cells $(\mathrm{C})$ in the growth plates of loaded tibias with and without SOCs loaded vertically (VL) and angularly (AL). RZ\&HZ, resting zone \& proliferative zone, HZ, hypertrophic zone. "tibia with SOC" and "tibia without SOC" refer to tibias from 30-dayold mice and 10-day-old rats respectively (see Figure S3A-B). Data are means \pm SD, two-tailed

1403 Student's $t$ test. In (A-C), $n=3$. 
1404

1405

1406

1407

1408

1409

1410

1411

1412

1413

1414

1415

1416

1417

1418

1419

1420
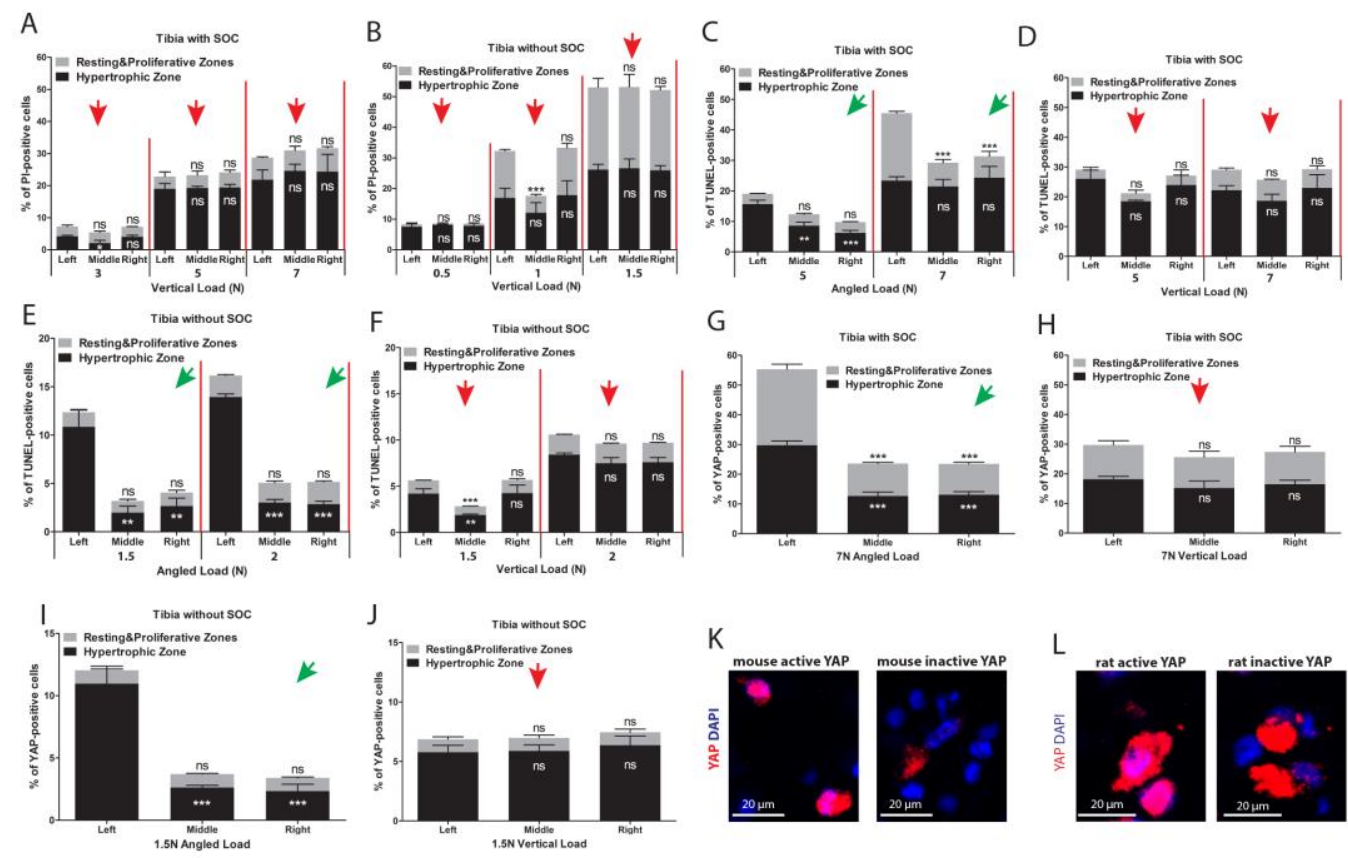

K
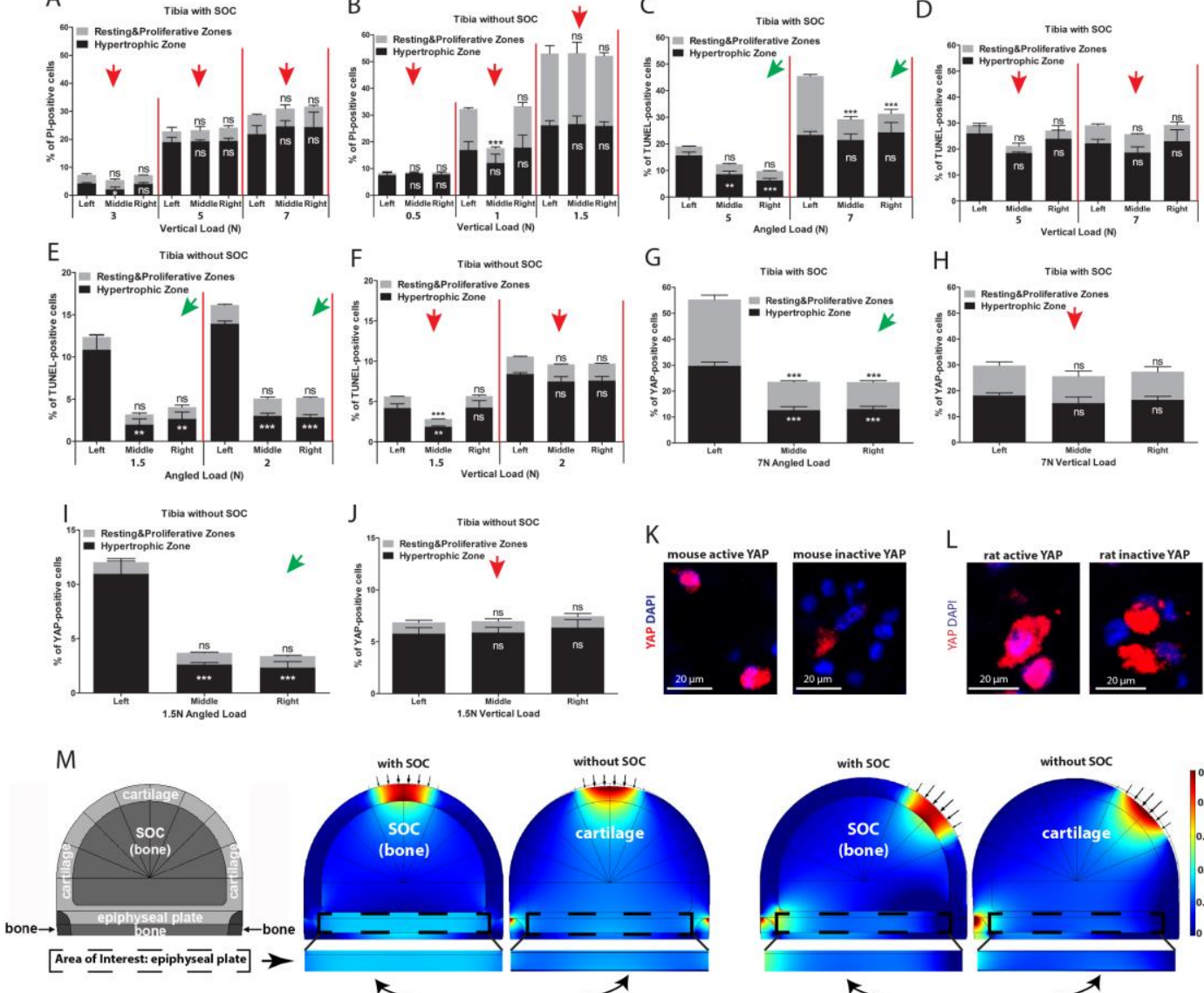

[Area of Interesti epiphyseal plate]

K
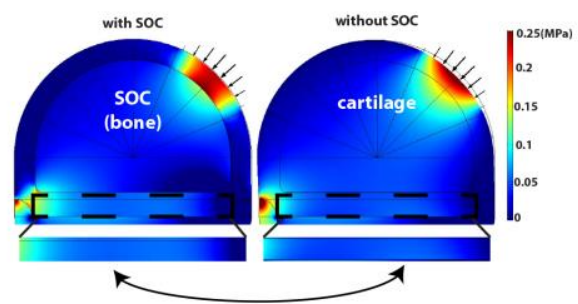

Figure 9-figure supplement 6. (A-B) Quantification of PI-positive chondrocytes in relation to lateral-medial direction of the growth plate of tibia loaded vertically with (A) and without (B) an SOC (see Figure 4A for details). (C-F) Quantification of TUNEL-positive chondrocytes in relation to lateral-medial direction of the growth plate of tibia with (C-D) and without (E-F) a SOC subjected to angular (C, E) and vertical (D, F) loading. (G-J) Quantification of distribution of YAP-positive chondrocytes in relation to lateral-medial direction within the resting\&proliferative and hypertrophic zones of the growth plate of angularly $(\mathrm{G}, \mathrm{I})$ and vertically $(\mathrm{H}, \mathrm{J})$ loaded tibia with $(\mathrm{G}-\mathrm{H})$ and without $(\mathrm{I}-\mathrm{J})$ an SOC. Only nuclearized active YAP as demonstrated in $\mathrm{k}-\mathrm{l}$ was quantified. The red and green arrows indicate the direction of loading (see the legend to Figure $4 \mathrm{a}$ for more details). Data are means \pm SD, One-way ANOVA. **, $\mathrm{P}<0.001$ and ***, $\mathrm{P}<0.0001$. In (A-J), n=3. ns, not significant. The black and white asterisks indicate $\mathrm{p}$-values for the resting\&proliferative and hypertrophic zones, respectively. (K-L) Immunostaining of active (nuclear) and inactive YAP in mouse $(\mathrm{K})$ and rat $(\mathrm{L})$ chondrocytes. (M) Hydrostatic compressive stress on epiphyseal plates from FEA modelling with physiological loading level. The small arrows indicate the direction of loading and the twoheaded curved arrows indicate areas for comparison. 

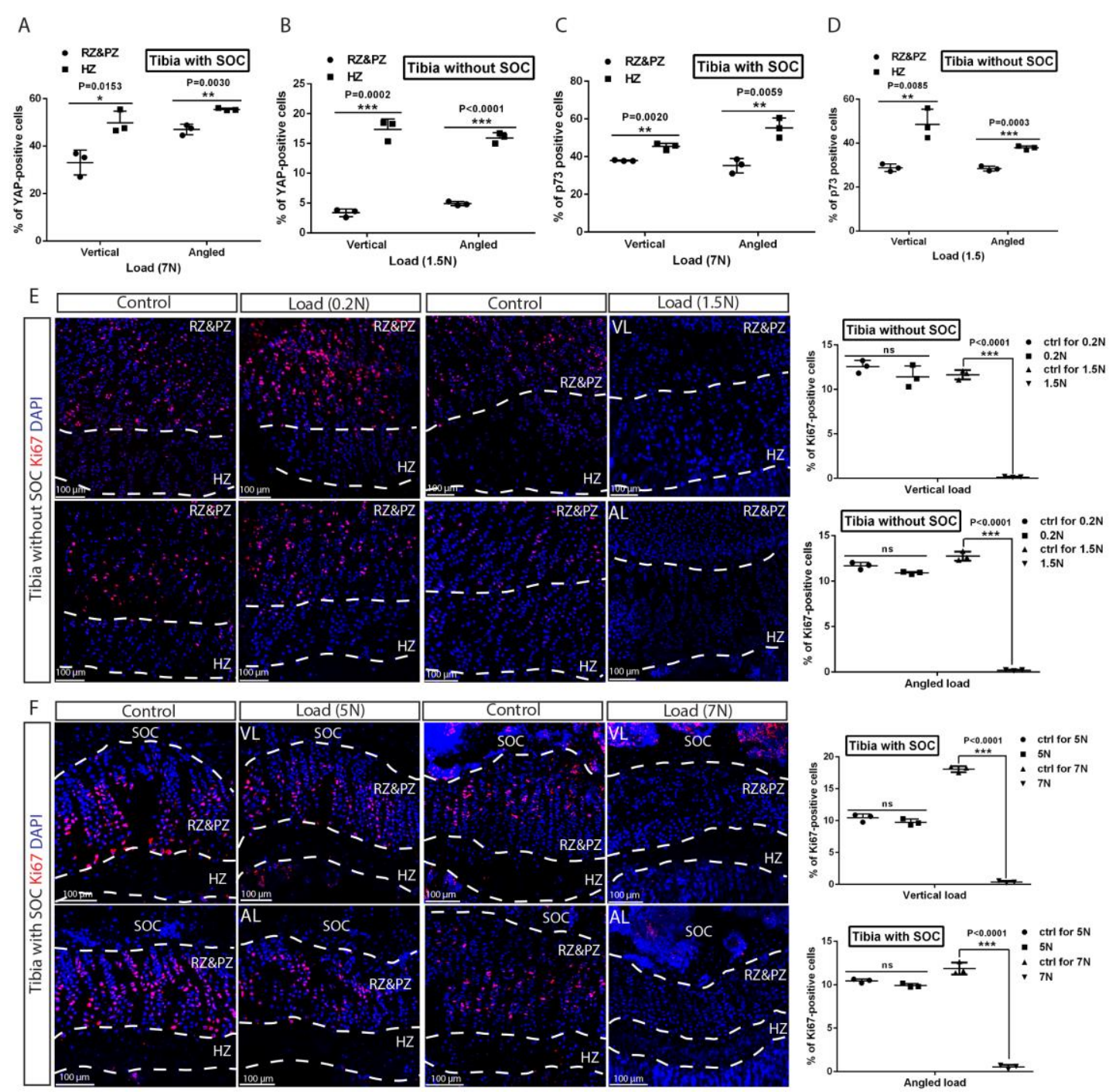

Figure 6-figure supplement 7. (A-D) Comparison of YAP (A-B) and p73 (C-D) staining between the resting plus proliferative zones (RZ\&PZ) and hypertrophic zone (HZ) in the growth plates of tibias with (A, C) and without (B, D) SOCs. (E-F) Representative images and quantification of the ki67 staining in the growth plates of tibias with (F) and without (E) SOCs. Data are means $\pm S D$, two-tailed Student's t test. In (A-F), n=3. ns, not significant. 


\section{Sham-operated Sciatic Nerve-resected}

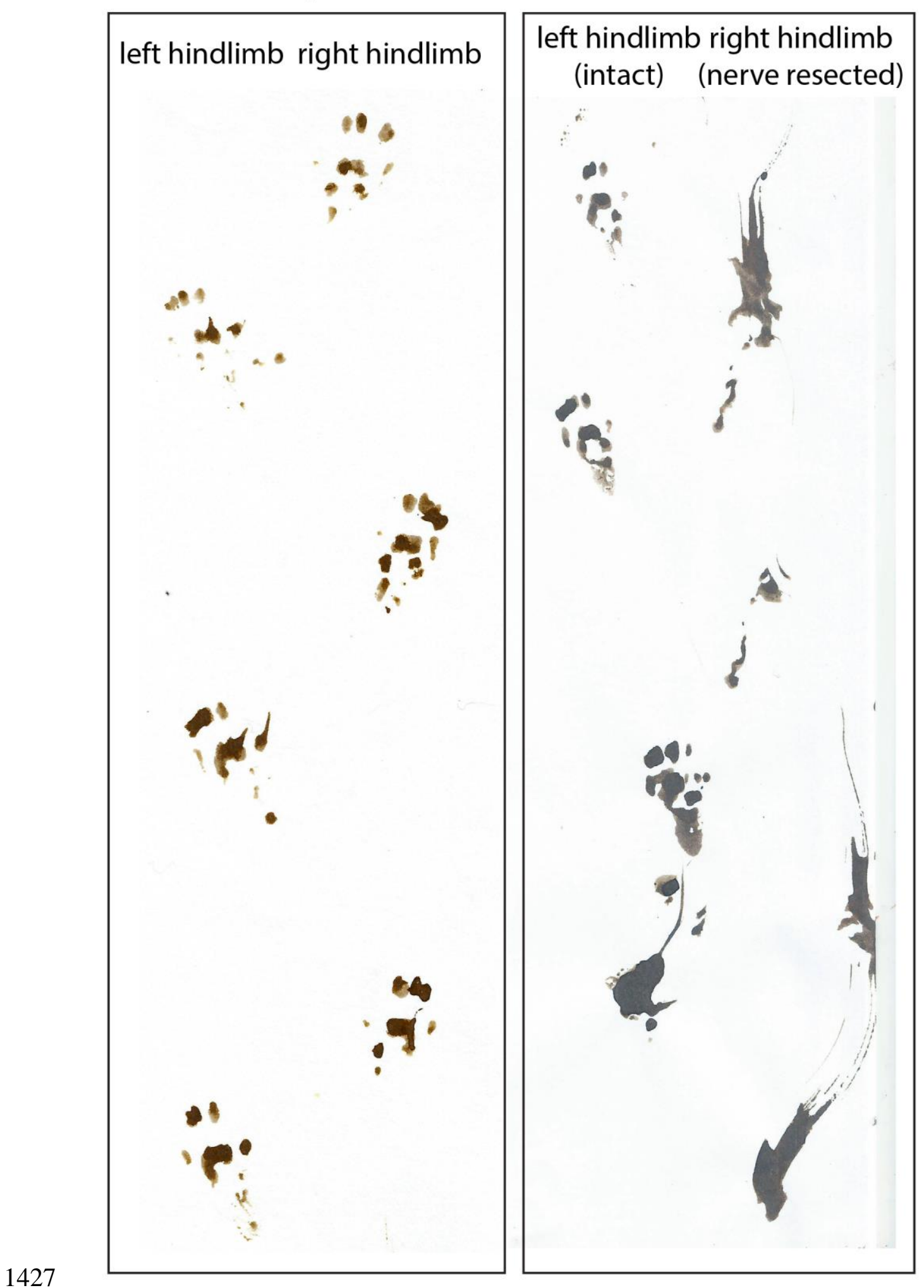

Figure 10-figure supplement 8. Footprint analysis revealed a clear difference between the right hindlimb of the sciatic nerve-resected and the sham-operated rats. 

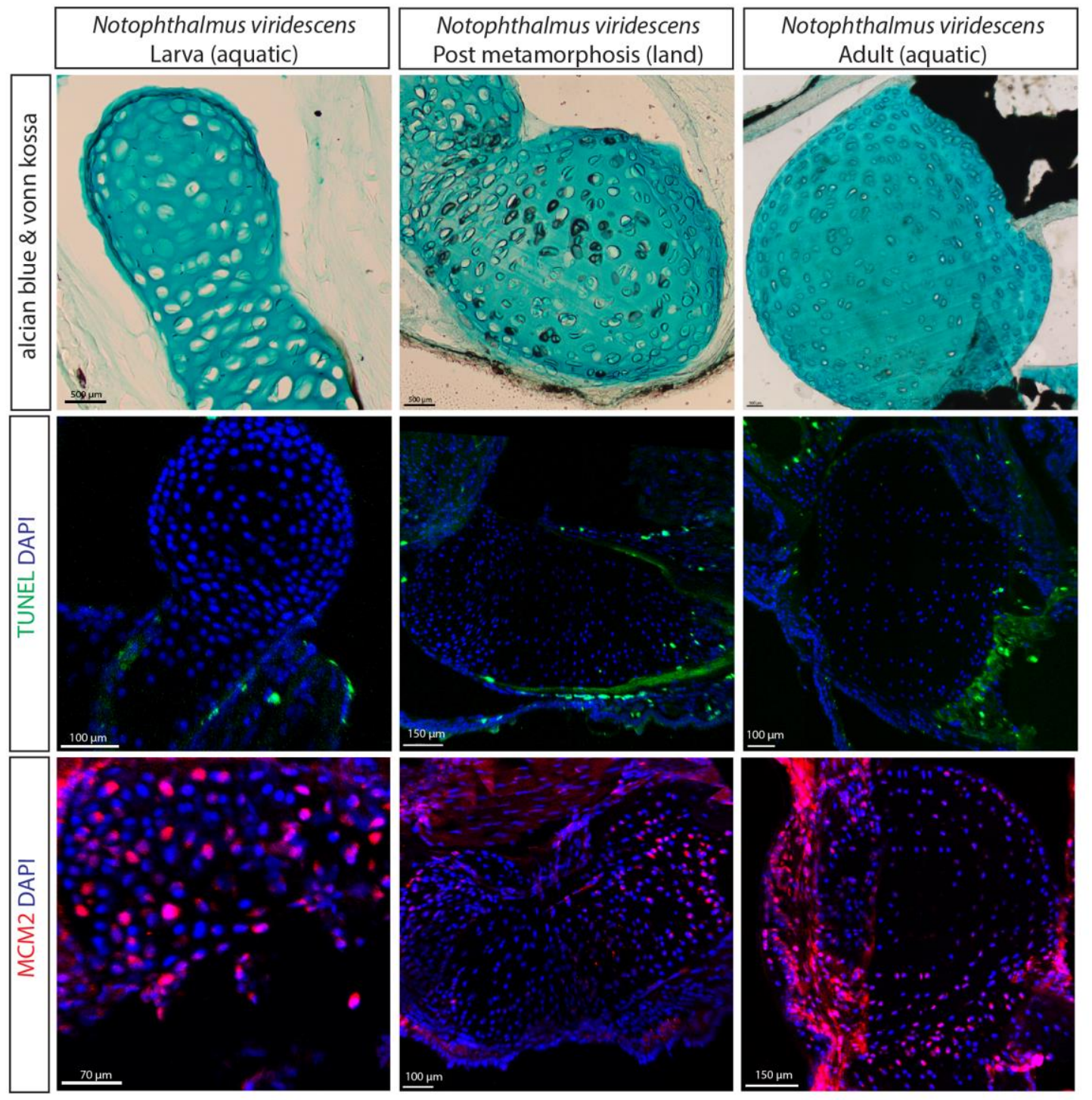

Figure 10-figure supplement 9. (A) Alcian blue and von kossa staining of the humerus of the newt (Notophthalmus viridescens) revealed calcified chondrocytes at the post metamorphosis land stage. (B-C) TUNEL (B) and DNA replication licensing factor MCM2 (C) staining of the same salamander species revealed no big differences in apoptotic and proliferating chondrocytes at the three developmental stages.

Video 1 3D model based on microCT surface images of a 1-3-day-old Pipistrellus pipistrellus s.l. (common pipistrelle bat). The age could not be determined more precisely.

1439 Video 2 Development of a gait pattern of Jerboa Jacuslus Jaculus.

1440 Video 3 Development of a gait pattern of Jerboa Jaculus Orientalis.

1441 Video 4 CT scan of the manus of a killer whale Orcinus orca.

1442 Supplementary File 1 Primary data on the level of ossification of the individual bones of all 1443 species of Chiroptera analyzed. 